\title{
Dynamics Research on Grouping Characteristics of a Shield Tunneling Machine's Thrust System
}

\author{
Yong Zhao ${ }^{\mathrm{a}, \mathrm{b}, ~ *}$, Hao Pan ${ }^{\mathrm{b}}$, Hao Wang ${ }^{\mathrm{a}, \mathrm{b}}$, Haidong Yu ${ }^{\mathrm{a}, \mathrm{b}}$ \\ ${ }^{a}$ State Key Laboratory of Mechanical System and Vibration, Shanghai Jiao Tong University \\ ${ }^{\mathrm{b}}$ Shanghai Key Laboratory of Digital Manufacture for Thin-walled Structures, Shanghai Jiao Tong University \\ 800 Dongchuan Road, Minhang District, Shanghai, 200240, P. R. China
}

\begin{abstract}
Grouped operation of a thrust system has a great effect on the performance of a shield tunneling machine. With consideration of the kinematic constraints from surrounding composite strata, the dynamic model of a grouped thrust system is firstly proposed to investigate the force transmission relationship between external resistances and driving forces. Then, the resistances model is derived based on the parametrized expression of two-layer composite strata. And the characteristics of each grouping mode for the thrust system are finally studied with variable geologic conditions by an index revealing the overload on segments. Results show that the contours of this index against impacting accelerations are a cluster of quasi-ellipses whose center can help to obtain the optimum grouping mode. Moreover, the adaptability of the thrust system to impacts will change significantly with the variations of the surrounding strata. These findings can serve as helpful instructions for the selection of grouping strategies in future tunneling projects.
\end{abstract}

Key Words: Shield Tunneling Machine; Dynamic Force Transmission; Grouping Strategy; Thrust System.

\section{Introduction}

The first successful implementation of Earth Pressure Balance (EPB) shield tunneling in Japan in late 1970s has led to the rapid development of this automated tunneling technique. Due to its higher working speed and better safety assurance, shield tunneling machine is now commonly used for tunnel constructions [1]. Recently, with the sharply increasing demand for urban underground infrastructures and public transportation systems such as metros, railways, etc., the shield tunneling

Corresponding author. Tel.: +86 2134206288

E-mail address: zhaoyong@ @jtu.edu.cn. 
technique has become one of the most attractive research topics in the field of underground space technology. A typical shield tunneling machine is generally comprised of a cutter, a thrust device and segments [2]. The thrust device is the most important component for providing sufficient feeding force to cut soils and rocks, which is mainly composed of dozens of parallel-arranged hydraulic cylinders.

In tunneling projects, shield tunneling machines always work in a narrow workspace with high temperature, large gravels, etc. Hence, accurate performance analysis for a shield tunneling machine has become a primary investigation prior to a successful tunnel construction. At present, performance analysis based on statistical models is a most prevalent approach. Within these models [3-6], measured geologic parameters and performance parameters were correspondingly converted into a database to carry out regression analysis. And the results were then used to predict the performance of the shield tunneling machine in the upcoming constructing section, together with initially detected geologic parameters. However, this technique regarded the performance analysis of a shield tunneling machine as a pure mathematical issue. The specific configurations for the thrust device of the machine and its interaction with the surrounding strata were ignored, which most likely led to constructing failures in tunneling along a curvilinear path, such as snake-like motion, being stuck, etc.

Additionally, in order to reduce the complexity of operation and control in practical implementations, grouped-control scheme is always applied to the hydraulic thrust device of a shield tunneling machine [7]. Due to the fact that the resistant forces, the bending moments and the torques acting on the cutter are always changing with the variation of surrounding strata, different grouping configurations of the mechanical structures will result in various ability to rectifying deviations and bearing impacts. As a result, load calculation and mechanism modeling become two necessary components of performance analysis for a shield tunneling machine.

A shield tunneling machine can be regarded as an underground robotic manipulator [8] and most current researches about the analysis of its thrust system is mainly based on theories of parallel mechanisms with actuation redundancy. Li et al. [9] proposed a general nonlinear time-varying dynamic model and analyzed the effect of physical parameters such as gear backlash and transmission error on the mechanical performance of the thrust system with their model. Both Liu et al. [10] and Deng et al. [11] presented a force transmission model based on Jacobian matrix 
for the redundant-actuated thrust system of the shield tunneling machine and conducted the selection for the optimum grouping strategy according to an index on the thrust force's variation. One of the most significant research findings is the force transmission model proposed by Deng et al. In [12], the force ellipse's eccentricity the thrust system was investigated based on Lagrange Function to analyze the force transmission characteristics with non-equidistant arrangements of cylinders. And in [13] a spatial deformation ellipse model was presented to study the variable stiffness-deformation characteristics of a shield tunneling machine, aiming to find better layout configurations for its thrust system. In [14] the layout arrangement of the jacks was optimized based on the force transmission spatial ellipse aiming to realize even application of forces on rear segments.

Load calculation can generally be realized in two ways: experimental measurement and theoretical model. Due to the complexity of tunneling experiment, theoretical analysis on thrust load is more frequently used in the field of shield tunneling research. The dynamic load model presented by Sugimoto et al. $[15,16]$ is accepted as the first comprehensive theoretical model, where several key construction parameters such as the excavated area and the cutter head's rotation were included in load calculation. Afterwards, improved theoretical models were gradually developed and becoming prevalent. Wang et al. [17] analyzed the roughness of the results from an empirical load equation and proposed a novel composite model whose accuracy could be improved by experimental data and further theoretical analysis. On the basis of empirical load models, Ates et al. [18] investigated the statistical correlations between TBM diameter and excavation capacity with a database of TBMs with various design parameters and thus presented a TBM selection approach that was tested in Turkish tunneling projects. Load analysis based on contact stress presented by Zhang et al. in [19] was a sufficiently accurate model for load prediction and an improved model was established to calculate the excavating load for soil-rock interbedded strata. In [20], theoretical analysis of tangential load and normal load was conducted to be compared with in-situ data, validating the correctness of the original model.

According to the above investigation, our research will concentrate on three aspects of improvements on the existing findings of force transmission model and excavating load calculation. Firstly, all the force transmission models were deduced in terms of static force balance without consideration of dynamic characteristics in 
tunneling projects. Secondly, constraints which restricted a shield tunneling machine into 3-DOF (degree of freedom) motion were not analyzed. And lastly, the bending moments acting on the cutter head in composite strata were seldom analyzed in existing load models.

In the current paper, the force transmission model between the driving forces in the hydro-cylinders and the external forces is studied, and the necessary cutting loads and surrounding resistant forces are calculated with regard to the characteristic parameters of composite strata. According to the mean square error of the driving forces in hydro-cylinders, an index on force distribution uniformity is then proposed to evaluate the grouping strategy of a thrust system. Finally, in searching for optimum performances, the grouping strategy of a shield tunneling machine in curvilinear tunneling projects under impacts is investigated with numerical simulation, which can serve as helpful instructions for the grouping mode design of future thrust systems.

\section{Dynamics Model of Thrust System}

\subsection{Analysis on the mechanism of thrust system}

The motions of a shield tunneling machine are restricted in a quasi-cylinder by constraint forces from surrounding strata:

1) The vertical motion of the shield tunneling machine perpendicular to the excavating axis is restricted by the upper and lower stratum;

2) The lateral motion of the shield tunneling machine perpendicular to the excavating axis is restricted by the left and right stratum;

3) The rotating motion of the shield tunneling machine along its own symmetry axis is restricted by the circumferential friction between the surrounding strata and the shield.

Therefore, a working shield tunneling machine contains only 3 independent motions: propelling, yawing and pitching. Due to the fact that the constraint forces are enough to prevent relative movements, the restrictions from the surrounding strata can be converted into a massless passive PU chain (Fig. 1), where P denotes a prismatic joint and $\mathrm{U}$ denotes a universal joint. 


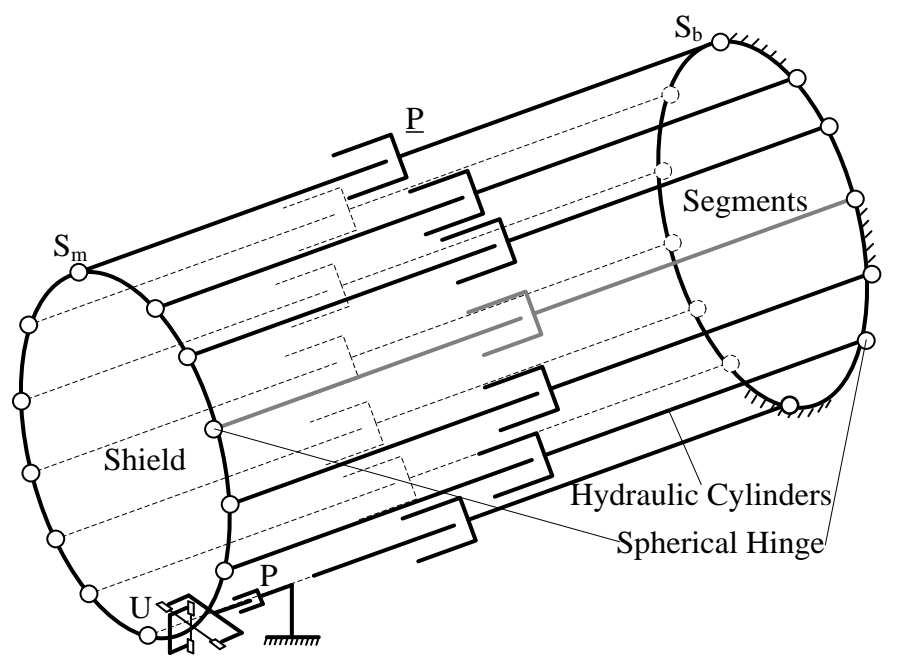

Fig.1. A schematic diagram of a shield tunneling machine's thrust system

Because shield tunneling is a dynamic process, the position and direction of the constraint chain will change with the movement of the cutter head. In our model, the junction point which connects the cutter head and the prismatic joint is coincident with the center of the universal joint and thus the displacement of the prismatic joint is tiny. Additionally, the position of the universal joint is related to the direction of a composite rotation and the direction of the axis of the prismatic joint is always consistent with the normal of the cutter head.

Moreover, the thrust device of a shield tunneling machine consists of $n$ parallel-arranged hydro-cylinders, which are jointed to the cutter head and the segments with spherical hinges. As a result, the thrust system, composed of the cutter head, the segments and the cylinders, is possessed with the essential characteristics of a redundantly actuated $n$-S $\underline{P} S$ parallel mechanism with 6-DOF spatial motion, where $S$ denotes a spherical hinge and $\underline{P}$ an actuated prismatic joint [2]. Thus, with consideration of the equivalent constraint chain, the thrust system can be regarded as a redundantly actuated $\mathrm{n}$-S $\underline{P} S / \mathrm{PU}$ parallel mechanism, whose schematic diagram is shown in Fig. 1.

\subsection{Kinematic analysis of thrust system}

Within the thrust system, the segments are directly mounted to the finished tunnel and thus act as the fixed platform, while the cutter head acts as the moving platform. Fig. 2 shows a schematic diagram of the thrust system at a random position 
and posture. An inertial coordinate system $O_{\mathrm{b}}-x_{\mathrm{b}} y_{\mathrm{b}} z_{\mathrm{b}}$ is established at the center of the fixed platform and a reference coordinate system $O_{\mathrm{m}}-x_{\mathrm{m}} y_{\mathrm{m}} z_{\mathrm{m}}$ is established at the center of the moving platform. SPS kinematic chains are branches without any constraint and therefore the motion of the moving platform is completely restricted by the passive PU chain, which means that the moving platform can only translate along $z$ axis with a variable $l$ and rotate around $x$ and $y$ axis respectively with variables $\alpha$ and $\beta$. According to vector triangles, the position vector of the moving platform can be derived as

$$
r_{\mathrm{m}}=\boldsymbol{r}_{\mathrm{PU}}^{\mathrm{b}}+\boldsymbol{l}_{\mathrm{P}}-\boldsymbol{\rho}_{\mathrm{U}}+\boldsymbol{l}
$$

where $r_{\mathrm{PU}}^{\mathrm{b}}$ denotes the position vector of the corresponding point of the PU chain on the fixed platform, $l_{\mathrm{p}}$ denotes the displacement vector between the prismatic joint and its corresponding point on the fixed platform, $\rho_{U}$ denotes the vector of the universal joint on the moving platform, $l$ denotes the displacement vector between the fixed end of the prismatic joint and the center of the universal joint.

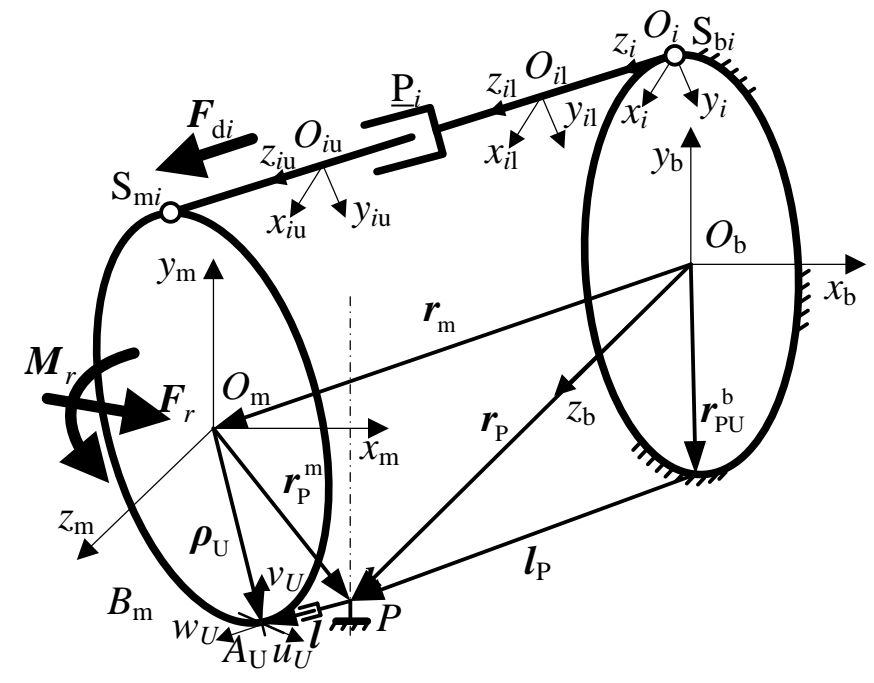

Fig.2. A schematic diagram of the displacement and rotation of the shield

In the reference coordinate system of the moving platform, the vector of the universal joint can be characterized as

$$
\boldsymbol{\rho}_{\mathrm{U}}^{\mathrm{m}}=\left(\begin{array}{lll}
R_{\mathrm{sh}} \cos \chi & R_{\mathrm{sh}} \sin \chi & 0
\end{array}\right)^{\mathrm{T}}
$$

where $\chi$ denotes the position of the universal joint in the reference coordinate system of the moving platform, the displacement vector between the fixed end of the prismatic joint and the center of the universal joint can be characterized as 
Given that the orientation cosine matrix for the prismatic joint of the PU chain relative to the moving platform and that for the moving platform relative to the fixed platform are respectively

$$
\begin{aligned}
& \boldsymbol{A}_{l}^{\mathrm{m}}=\left(\begin{array}{ccc}
\cos (\chi+\pi / 2) & -\sin (\chi+\pi / 2) & 0 \\
\sin (\chi+\pi / 2) & \cos (\chi+\pi / 2) & 0 \\
0 & 0 & 1
\end{array}\right) \\
& \boldsymbol{A}_{\mathrm{m}}=\left(\begin{array}{ccc}
\cos \beta & 0 & \sin \beta \\
\sin \alpha \sin \beta & \cos \alpha & -\sin \alpha \cos \beta \\
-\cos \alpha \sin \beta & \sin \alpha & \cos \alpha \cos \beta
\end{array}\right)
\end{aligned}
$$

The following equations can be derived

$$
\begin{gathered}
\boldsymbol{\rho}_{\mathrm{U}}=\boldsymbol{A}_{\mathrm{m}} \boldsymbol{\rho}_{\mathrm{U}}^{\mathrm{m}}=\left(\begin{array}{c}
R_{\mathrm{sh}} \cos \chi \cos \beta \\
R_{\mathrm{sh}} \cos \chi \sin \alpha \sin \beta+R_{\mathrm{sh}} \sin \chi \cos \alpha \\
-R_{\mathrm{sh}} \cos \chi \cos \alpha \sin \beta+R_{\mathrm{sh}} \sin \chi \sin \alpha
\end{array}\right) \\
\boldsymbol{l}=\boldsymbol{A}_{\mathrm{m}} \boldsymbol{A}_{l}^{\mathrm{m}} \boldsymbol{l}^{\mathrm{m}}=\left(\begin{array}{c}
l \sin \beta \\
-l \sin \alpha \cos \beta \\
l \cos \alpha \cos \beta
\end{array}\right)
\end{gathered}
$$

Thus, the angular acceleration of the moving platform can be derived using the derivate of $\boldsymbol{\omega}_{\mathrm{m}}$ with consideration that $\hat{\boldsymbol{\omega}}_{\mathrm{m}}=\boldsymbol{A}_{\mathrm{m}}^{\mathrm{T}} \dot{\boldsymbol{m}}_{\mathrm{m}}$; the translational acceleration of that can be derived using the second-order derivate of $r_{\mathrm{m}}$ with Eq. (6) and (7); and a system of kinematic equations characterized by coordinates $\left(\begin{array}{lll}l & \alpha & \beta\end{array}\right)$ can then be derived to describe the 3-DOF motion of the moving platform.

However, among the kinematic coordinates aforementioned, the length of the prismatic joint $l$ cannot reflect the actual motion of cutter head. Due to the fact that the movement of cutter head relative to the prismatic joint is extremely small, it's reasonable to assume that the radius of the center of the moving platform $r \approx l_{\mathrm{p}}+l$. During each tunneling step, $l_{\mathrm{p}}$ is a constant and thus we can obtain $\dot{r} \approx \dot{i}$ and $\ddot{r} \approx \ddot{l}$. Therefore, the accelerations of the moving platform in the expression of the generalized coordinates $\boldsymbol{q}=\left(\begin{array}{llll}r & \alpha & \beta\end{array}\right)^{\mathrm{T}} \quad$ can be derived as

$$
\begin{gathered}
\dot{\boldsymbol{\omega}}_{\mathrm{m}}=\boldsymbol{J}_{\mathrm{Rm}} \ddot{\boldsymbol{q}}+\boldsymbol{\sigma} \\
\ddot{\boldsymbol{r}}_{\mathrm{m}}=\boldsymbol{J}_{\mathrm{Tm}} \ddot{\boldsymbol{q}}+\boldsymbol{\varepsilon}
\end{gathered}
$$

where (see Appendix A and B for details of each element)

$$
\boldsymbol{J}_{\mathrm{Rm}}=\left(\begin{array}{ccc}
0 & 1 & 0 \\
0 & 0 & \cos \alpha \\
0 & 0 & \sin \alpha
\end{array}\right) \quad \boldsymbol{\sigma}=\left(\begin{array}{c}
0 \\
-\sin \alpha \dot{\alpha} \dot{\beta} \\
\cos \alpha \dot{\alpha} \dot{\beta}
\end{array}\right)
$$




$$
\boldsymbol{J}_{\mathrm{Tm}}=\left(\begin{array}{lll}
J_{\mathrm{Tm} 11} & J_{\mathrm{Tm} 12} & J_{\mathrm{Tm} 13} \\
J_{\mathrm{Tm} 21} & J_{\mathrm{T} m 22} & J_{\mathrm{Tm} 23} \\
J_{\mathrm{Tm} 31} & J_{\mathrm{T} m 2} & J_{\mathrm{Tm} 33}
\end{array}\right) \quad \boldsymbol{\varepsilon}=\left(\begin{array}{c}
\varepsilon_{11} \\
\varepsilon_{21} \\
\varepsilon_{31}
\end{array}\right)
$$

Because the SPS chain has a partial freedom of rotation, the spherical hinge that connects the prismatic joint and the fixed platform can be replaced with a universal joint during kinematic analysis. Thus, each branch is converted into a UPS chain. A single kinematic chain of the thrust system and the constraint chain form a closed loop, which means the vector characterizing the $i_{\text {th }}$ chain can be presented as

$$
\boldsymbol{l}_{i}=\boldsymbol{l}_{\mathrm{P}}+\boldsymbol{l}+\boldsymbol{\rho}_{\mathrm{PU}}^{\mathrm{b}}-\boldsymbol{\rho}_{\mathrm{B} i}+\boldsymbol{\rho}_{\mathrm{P} i}-\boldsymbol{\rho}_{\mathrm{U}}
$$

where $\rho_{\mathrm{PU}}^{\mathrm{b}}$ is the body hinge vector of the corresponding point on the fixed, $\rho_{\mathrm{B} i}$ is the body hinge vector of the $i_{\text {th }}$ universal joint on the fixed platform, $\rho_{\mathrm{P} i}$ is the body hinge vector of the $i_{\text {th }}$ spherical hinge of the moving platform; details for all the elements of $l_{i}$ can be found in Appendix C.

Thus, the unit vector of the $i_{\text {th }}$ S $\underline{P} S$ chain can be derived as

$$
\boldsymbol{u}_{i}=\frac{\boldsymbol{l}_{i}}{\left|\boldsymbol{l}_{i}\right|}
$$

Based on the reference coordinate system connected to the $i_{\text {th }}$ SPS chain (Fig. 2), its orientation cosine matrix can be deduced with $\eta_{i}$ (the rotating angle along the $x_{i}$ axis), $\phi_{i}$ (the rotating angle about the $y_{i}$ axis) and $\varphi_{i}$ (the angle reflecting the fixing orientation of the $i_{\text {th }}$ universal joint). The corresponding orientation cosine matrix for these three rotations are listed respectively as

$$
\boldsymbol{A}_{\phi_{i}}=\left(\begin{array}{ccc}
\cos \phi_{i} & 0 & \sin \phi_{i} \\
0 & 1 & 0 \\
-\sin \phi_{i} & 0 & \cos \phi_{i}
\end{array}\right) \quad \boldsymbol{A}_{\eta_{i}}=\left(\begin{array}{ccc}
1 & 0 & 0 \\
0 & \cos \eta_{i} & -\sin \eta_{i} \\
0 & \sin \eta_{i} & \cos \eta_{i}
\end{array}\right) \quad \boldsymbol{A}_{\varphi_{i}}=\left(\begin{array}{ccc}
\cos \varphi_{i} & -\sin \varphi_{i} & 0 \\
\sin \varphi_{i} & \cos \varphi_{i} & 0 \\
0 & 0 & 1
\end{array}\right)
$$

Accordingly, the transformation equation for the unit vector of the $i_{\text {th }}$ S $\underline{S}$ s chain can be derived as

$$
\boldsymbol{A}_{i} \boldsymbol{u}_{i}^{i}=\boldsymbol{u}_{i}
$$

where $\boldsymbol{u}_{i}^{i}=\left(\begin{array}{lll}0 & 0 & 1\end{array}\right)^{\mathrm{T}}, \quad \boldsymbol{A}_{i}=\boldsymbol{A}_{\varphi_{i}} \boldsymbol{A}_{\phi_{i}} \boldsymbol{A}_{\eta_{i}}$. Thus, the rotating angles of the $i_{\text {th }}$ S $\underline{P} S$ chain can be calculated with the following equation

$$
\left(\begin{array}{c}
\sin \phi_{i} \cos \eta_{i} \\
-\sin \eta_{i} \\
\cos \phi_{i} \cos \eta_{i}
\end{array}\right)=\boldsymbol{A}_{\varphi_{i}}^{\mathrm{T}} \boldsymbol{u}_{i}
$$

Finally, with the calculation of the kinematics of spherical hinges, the translational acceleration of the $i_{\text {th }}$ spherical hinge on the moving platform can be derived as 


$$
\ddot{\boldsymbol{r}}_{\mathrm{P} i}=\boldsymbol{\delta}_{\mathrm{P} i} \ddot{\boldsymbol{q}}+\boldsymbol{\zeta}_{\mathrm{P} i}
$$

where $\boldsymbol{\delta}_{\mathrm{P} i}=\boldsymbol{J}_{\mathrm{Tm}}-\boldsymbol{\rho}_{\mathrm{P} i} \boldsymbol{J}_{\mathrm{Rm}}, \quad \boldsymbol{\zeta}_{\mathrm{P} i}=\boldsymbol{\varepsilon}-\boldsymbol{\rho}_{\mathrm{P} i} \boldsymbol{\sigma}-\boldsymbol{\omega}_{\mathrm{m}} \boldsymbol{\rho}_{\mathrm{P} i} \boldsymbol{\omega}_{\mathrm{m}}$.

Two reference coordinate systems $O_{i \mathrm{u}}-x_{i \mathrm{u}} y_{i \mathrm{u}} z_{i \mathrm{ul}}$ and $O_{i 1}-x_{i 1} y_{i 1} z_{i l}$ are then respectively established at the mass center of the $i_{\text {th }}$ upper link and that of the $i_{\text {th }}$ lower link (Fig. 2). According to the vector relation between these mass centers and the centers of the $i_{\text {th }}$ spherical hinge as well as the $i_{\text {th }}$ universal joint, the kinematics of the $i_{\text {th }}$ upper link and that of the $i_{\text {th }}$ lower link in the expression of the generalized coordinates $q$ can be respectively derived as

$$
\begin{gathered}
\dot{\boldsymbol{\omega}}_{i \mathrm{u}}=\boldsymbol{J}_{\mathrm{R} i \mathrm{u}} \ddot{\boldsymbol{q}}+\boldsymbol{\sigma}_{i \mathrm{u}} \\
\ddot{\boldsymbol{r}}_{i \mathrm{u}}=\boldsymbol{J}_{\mathrm{T} i \mathrm{q}} \ddot{\boldsymbol{q}}+\boldsymbol{\varepsilon}_{i \mathrm{u}} \\
\dot{\boldsymbol{\omega}}_{i \mathrm{l}}=\boldsymbol{J}_{\mathrm{R} i \mathrm{l}} \ddot{\boldsymbol{q}}+\boldsymbol{\sigma}_{i 1} \\
\ddot{\boldsymbol{r}}_{i 1}=\boldsymbol{J}_{\mathrm{T} \mathrm{i}} \ddot{\boldsymbol{q}}+\boldsymbol{\varepsilon}_{i 1}
\end{gathered}
$$

where

$$
\begin{gathered}
\boldsymbol{J}_{\mathrm{T} i \mathrm{u}}=\boldsymbol{\delta}_{\mathrm{P} i}+\frac{\boldsymbol{\rho}_{i \mathrm{u}} \boldsymbol{u}_{i} \delta_{\mathrm{P} i}}{\left|\boldsymbol{l}_{i}\right|} \quad \boldsymbol{J}_{\mathrm{T} i \mathrm{l}}=\frac{\boldsymbol{\rho}_{i \mathrm{u}} \boldsymbol{u}_{i} \boldsymbol{\delta}_{\mathrm{P} i}}{\left|\boldsymbol{l}_{i}\right|} \\
\boldsymbol{\varepsilon}_{i \mathrm{u}}=\left(\boldsymbol{u}_{i} \dot{\boldsymbol{u}}_{i}\right) \times\left(\boldsymbol{\rho}_{i \mathrm{u}} \boldsymbol{u}_{i} \dot{\boldsymbol{u}}_{i}\right)+\frac{\boldsymbol{\rho}_{i \mathrm{u}} \boldsymbol{u}_{i} \boldsymbol{\zeta}_{\mathrm{P} i}-2\left|\boldsymbol{i}_{i}\right| \boldsymbol{\rho}_{i \mathrm{u}} \boldsymbol{u}_{i} \dot{\boldsymbol{u}}_{i}}{\left|\boldsymbol{t}_{i}\right|}+\boldsymbol{\zeta}_{\mathrm{P} i} \\
\boldsymbol{\varepsilon}_{i \mathrm{l}}=\left(\boldsymbol{u}_{i} \dot{\boldsymbol{u}}_{i}\right) \times\left(\boldsymbol{\rho}_{i \mathrm{l}} \boldsymbol{u}_{i} \dot{\boldsymbol{u}}_{i}\right)+\frac{\boldsymbol{\rho}_{i 1} \boldsymbol{u}_{i} \boldsymbol{\zeta}_{\mathrm{P} i}-2\left|\boldsymbol{i}_{i}\right|_{i} \boldsymbol{\rho}_{i 1} \boldsymbol{u}_{i} \dot{\boldsymbol{u}}_{i}}{\left|\boldsymbol{l}_{i}\right|} \\
\boldsymbol{J}_{\mathrm{R} i \mathrm{u}}=\boldsymbol{J}_{\mathrm{R} i \mathrm{l}}=\frac{\boldsymbol{u}_{i} \boldsymbol{\delta}_{\mathrm{P} i}}{\left|\boldsymbol{l}_{i}\right|} \quad \boldsymbol{\sigma}_{i \mathrm{u}}=\boldsymbol{\sigma}_{i \mathrm{l}}=\frac{\boldsymbol{u}_{i} \zeta_{\mathrm{P} i}-2\left|\boldsymbol{i}_{i}\right| \boldsymbol{u}_{i} \dot{\boldsymbol{u}}_{i}}{\left|\boldsymbol{l}_{i}\right|}
\end{gathered}
$$

\subsection{Dynamic analysis of thrust system}

According to Newton-Euler formulae, the dynamic equations of the thrust system can be derived as

$$
\begin{gathered}
\boldsymbol{m}_{\mathrm{m}} \ddot{\boldsymbol{r}}_{m}=\boldsymbol{F}_{\mathrm{m}}^{\mathrm{c}}+\boldsymbol{F}_{\mathrm{m}}^{\mathrm{w}} \\
\boldsymbol{I}_{\mathrm{m}} \dot{\boldsymbol{\omega}}_{\mathrm{m}}+\boldsymbol{\omega}_{\mathrm{m}} \times\left(\boldsymbol{I}_{\mathrm{m}} \boldsymbol{\omega}_{\mathrm{m}}\right)=\boldsymbol{M}_{\mathrm{m}}^{\mathrm{c}}+\boldsymbol{M}_{\mathrm{m}}^{\mathrm{w}} \\
\boldsymbol{m}_{i \mathrm{u}} \ddot{\boldsymbol{r}}_{i \mathrm{u}}=\boldsymbol{F}_{i \mathrm{u}}^{\mathrm{c}}+\boldsymbol{F}_{i \mathrm{u}}^{\mathrm{w}} \\
\boldsymbol{I}_{i \mathrm{u}} \dot{\boldsymbol{\omega}}_{i \mathrm{u}}+\boldsymbol{\omega}_{i \mathrm{u}} \times\left(\boldsymbol{I}_{i \mathrm{u}} \boldsymbol{\omega}_{i \mathrm{u}}\right)=\boldsymbol{M}_{i \mathrm{u}}^{\mathrm{c}}+\boldsymbol{M}_{i \mathrm{u}}^{\mathrm{w}} \\
\boldsymbol{m}_{i 1} \ddot{\boldsymbol{r}}_{i 1}=\boldsymbol{F}_{i 1}^{\mathrm{c}}+\boldsymbol{F}_{i 1}^{\mathrm{w}} \\
\boldsymbol{I}_{i 1} \dot{\boldsymbol{\omega}}_{i 1}+\boldsymbol{\omega}_{i 1} \times\left(\boldsymbol{I}_{i 1} \boldsymbol{\omega}_{i 1}\right)=\boldsymbol{M}_{i 1}^{\mathrm{c}}+\boldsymbol{M}_{i 1}^{\mathrm{w}}
\end{gathered}
$$


where $\boldsymbol{m}_{\mathrm{m}}, \boldsymbol{m}_{i \mathrm{u}}$ and $\boldsymbol{m}_{i 1}$ respectively denote the mass matrix of the shield, that of the piston rod and the cylinder of the $i_{\mathrm{th}}$ hydro-cylinder, $\boldsymbol{I}_{\mathrm{m}}, \boldsymbol{I}_{\mathrm{iu}}$ and $\boldsymbol{I}_{i 1}$ respectively denote the rotary inertia matrix of the shield, that of the piston rod and the cylinder of the $i_{\text {th }}$ hydro-cylinder, $\boldsymbol{F}_{\mathrm{m}}^{\mathrm{w}}, \boldsymbol{F}_{i \mathrm{u}}^{\mathrm{w}}, \boldsymbol{F}_{i 1}^{\mathrm{w}}$ and $\boldsymbol{M}_{\mathrm{m}}^{\mathrm{w}}, \boldsymbol{M}_{i \mathrm{u}}^{\mathrm{w}}, \boldsymbol{M}_{i 1}^{\mathrm{w}}$ denote the vector sum of external forces and that of external torques acting on the shield, the piston rod and the cylinder of the $i_{\text {th }}$ hydro-cylinder respectively, $\boldsymbol{F}_{\mathrm{m}}^{\mathrm{c}}, \boldsymbol{F}_{\mathrm{iu}}^{\mathrm{c}}, \boldsymbol{F}_{i 1}^{\mathrm{c}}$ and $\boldsymbol{M}_{\mathrm{m}}^{\mathrm{c}}, \boldsymbol{M}_{\mathrm{iu}}^{\mathrm{c}}, \boldsymbol{M}_{i 1}^{\mathrm{c}}$ denote the vector sum of the ideal constraint forces and torques from the moving joints acting on the shield, the piston rod and the cylinder of the $i_{\text {th }}$ hydro-cylinder respectively.

Regarding a working shield tunneling machine, the mass of each hydro-cylinder is extremely small and thus negligible when comparing with the shield, which means it is in static equilibrium. Therefore, the constraint force from an arbitrary spherical hinge acting on the shield is actually equal to the driving force in the corresponding hydro-cylinder. Then, with these static equilibrium equations and eliminating the ideal constraint forces with the principle of virtual work [21], the dynamic equations of the thrust system in Shiehlen's form can be derived as

$$
\boldsymbol{H}^{\mathrm{T}} \boldsymbol{\Phi} \boldsymbol{H} \ddot{\boldsymbol{q}}+\boldsymbol{H}^{\mathrm{T}} \boldsymbol{K}(\boldsymbol{q}, \dot{\boldsymbol{q}})=\boldsymbol{H}^{\mathrm{T}} \boldsymbol{F}^{\mathrm{w}}
$$

and all the vector matrices on the left side of the equation are illustrated as (see Appendix D for details of each element)

$$
\begin{gathered}
\boldsymbol{\Phi}=\left(\begin{array}{lll}
\boldsymbol{m}_{\mathrm{m}} & \\
& \boldsymbol{I}_{\mathrm{m}}
\end{array}\right) \quad \boldsymbol{H}=\left(\begin{array}{l}
\boldsymbol{J}_{\mathrm{Tm}} \\
\boldsymbol{J}_{\mathrm{Rm}}
\end{array}\right) \\
\boldsymbol{K}(\boldsymbol{q}, \dot{\boldsymbol{q}})=\left(\begin{array}{llllll}
K_{11} & K_{21} & K_{31} & K_{41} & K_{51} & K_{61}
\end{array}\right)^{\mathrm{T}}
\end{gathered}
$$

For a shield tunneling machine, the total external load $\boldsymbol{F}^{\mathrm{w}}$ mainly results from the resistance of surrounding strata $\boldsymbol{F}_{r e}$ and $\boldsymbol{M}_{r e}$, and the driving force of the hydro-cylinders $\boldsymbol{F}_{\mathrm{d} i}$ (Fig. 2). In the situation when each hydro-cylinder is independently controlled, the total driving force $\boldsymbol{F}_{\mathrm{d}}$ and its torque $\boldsymbol{M}_{\mathrm{d}}$ can be respectively presented with the following equations

$$
\begin{gathered}
\boldsymbol{F}_{\mathrm{d}}=\sum_{i} \boldsymbol{A}_{i} \boldsymbol{F}_{\mathrm{d} i} \\
\boldsymbol{M}_{\mathrm{d}}=\sum_{i} \boldsymbol{\rho}_{\mathrm{P} i} \times\left(\boldsymbol{A}_{i} \boldsymbol{F}_{\mathrm{d} i}\right)
\end{gathered}
$$

where $\boldsymbol{F}_{\mathrm{d} i}=\left(\begin{array}{llll}0 & 0 & f_{\mathrm{d} i}\end{array}\right)^{\mathrm{T}}$ denotes the driving force of the $i_{\text {th }}$ hydro-cylinder along its axis, $\rho_{\mathrm{P} i}=\boldsymbol{A}_{\mathrm{m}} \rho_{\mathrm{P} i}^{\mathrm{m}}$ and $\rho_{\mathrm{P} i}^{\mathrm{m}}$ denotes the body hinge vector of the $i_{\text {th }}$ spherical hinge on the 
shield in the reference coordinate system. Thus, through inserting Eqn. (26) and (27) into our dynamic model and using the $L_{2}$-norm optimizing method for redundant actuating loads [22], the mapping from the resistance to the driving load within the shield tunneling machine can be derived as

$$
\boldsymbol{f}^{\mathrm{d}}=-\boldsymbol{B}^{+} \boldsymbol{H}^{\mathrm{T}} \boldsymbol{F}^{\mathrm{r}}+\boldsymbol{B}^{+}\left[\boldsymbol{H}^{\mathrm{T}} \boldsymbol{\Phi} \boldsymbol{H} \ddot{\boldsymbol{q}}+\boldsymbol{H}^{\mathrm{T}} \boldsymbol{K}(\boldsymbol{q}, \dot{\boldsymbol{q}})\right]
$$

Where $\boldsymbol{f}^{\mathrm{d}}=\left(\begin{array}{llllll}f_{\mathrm{d} 1} & f_{\mathrm{d} 2} & \cdots & f_{\mathrm{d} i} & \cdots & \left.f_{\mathrm{d} n}\right)^{\mathrm{T}},\end{array},\left(\square^{+}\right.\right.$denotes a Moore-Penrose generalized inverse matrix and all the vector matrices are illustrated as (see Appendix D and E for details of each element)

$$
\begin{gathered}
\boldsymbol{F}^{\mathrm{r}}=\left(\begin{array}{lll}
\boldsymbol{F}_{r e} & \boldsymbol{M}_{r e}
\end{array}\right)^{\mathrm{T}} \\
\boldsymbol{B}=\left[\begin{array}{llllll}
\boldsymbol{B}_{1} & \boldsymbol{B}_{2} & \cdots & \boldsymbol{B}_{i} & \cdots & \boldsymbol{B}_{n}
\end{array}\right] \\
\boldsymbol{B}_{i}=\boldsymbol{H}^{\mathrm{T}}\left[\begin{array}{c}
\boldsymbol{A}_{i} \\
\boldsymbol{\rho}_{\mathrm{Pi}} \boldsymbol{A}_{i}
\end{array}\right] \boldsymbol{e}_{3}=\left(\begin{array}{llll}
B_{i 11} & B_{i 21} & B_{i 31}
\end{array}\right)^{\mathrm{T}}
\end{gathered}
$$

\subsection{Discussions about grouping's effect on the dynamics of thrust system}

In the practical operation of a shield tunneling machine, the steering angle of the shield is extremely small and the maximum propelling distance is relatively short. Hence, the normal of the cutter head is able to stay approximately in a line with the normal of the end face of the segments, which means each SPS chain is almost vertical to the base. Therefore, when analyzing the dynamics of a grouped thrust system, the paper deems that all the hydro-cylinders in each group can be simplified into equivalent one.

As a result, the thrust system is converted into an $n_{\mathrm{G}}-\mathrm{S} \underline{\mathrm{PS}}$ parallel mechanism, where $n_{\mathrm{G}}$ denotes the number of groups. Within each group of the equivalent mechanism, the position of the equivalent hydro-cylinder can be calculated using the arithmetic average value of the central angles of all the hydro-cylinders and its thrust can be derived as the sum of their propelling forces. Through substituting the elements related to the hydro-cylinders, the dynamic equations of the grouped thrust system can be derived in a similar form with that of the ungrouped thrust system.

\section{Analysis of Acting Resistances}

In tunneling projects, the three major resistances on the shield tunneling machine are respectively the penetration resistance, the earth pressure normal to the end face of 

the cutter head and the frictional force [17-19]. The penetration resistance is a dynamic load mainly related to the characteristics of cutting (propelling speed, rotating speed of the cutter head, strata characteristics, etc.) while the other two are mainly resulted from the interaction between the shield and the surrounding earth. Because the resistances acting on the shield tunneling machine are highly sensitive to the variation of the characteristics of surrounding strata, accurate analysis models for these resistances are very important to our investigation on the grouping strategy.

\subsection{Analysis on the penetration resistance}

In the situation of tunneling in composite strata, different cutters are often simultaneously mounted on the cutter head to deal with composite strata. Within our research, the cutter head is composed of 20 disc cutters and 64 scrapers that are arranged symmetrically about the axes (Fig. 3). According to the load model for scrapers in [23] and considering Rostami's load model for disc cutters [24], the total penetrating resistance acting on the cutter head can be derived as

$$
\begin{gathered}
F^{\mathrm{pen}}=\sum_{j=1}^{64} \frac{c_{j}^{\mathrm{coh}} s^{\mathrm{scp}}}{B_{j}^{\mathrm{scp}}}\left(\cos \theta^{\mathrm{scp}}-\mu_{j}^{\mathrm{scp}} \sin \theta^{\mathrm{scp}}\right)+\sum_{k=1}^{20} \lambda^{\mathrm{disc}} Q_{k}^{\mathrm{disc}} \frac{d^{\mathrm{disc}} w^{\mathrm{disc}} \theta^{\mathrm{disc}}}{2(\psi+1)} \cos \frac{\theta^{\mathrm{disc}}}{2} \\
\left\{\begin{array}{l}
B_{j}^{\mathrm{scp}}=\left(\cos \alpha^{\mathrm{scp}}-\mu_{j}^{\mathrm{R}} \sin \theta^{\mathrm{scp}}\right)\left(\sin \alpha^{\mathrm{scp}}+\mu_{j}^{\mathrm{scp}} \cos \theta^{\mathrm{scp}}\right)+\left(\cos \alpha^{\mathrm{scp}}-\mu_{j}^{\mathrm{scp}} \sin \theta^{\mathrm{scp}}\right)\left(\sin \alpha^{\mathrm{scp}}+\mu_{j}^{\mathrm{R}} \cos \theta^{\mathrm{scp}}\right) \\
Q_{k}^{\mathrm{disc}}=\sqrt[3]{l^{\mathrm{disc}}\left(\sigma_{k}^{\mathrm{c}}\right)^{2} \sigma_{k}^{\mathrm{t}} /\left(\theta^{\mathrm{disc}} \sqrt{d^{\mathrm{disc}} w^{\mathrm{disc}} / 2}\right)}
\end{array}\right.
\end{gathered}
$$

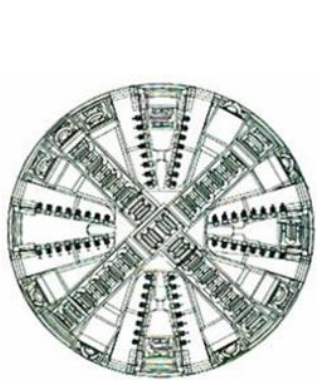

(a)

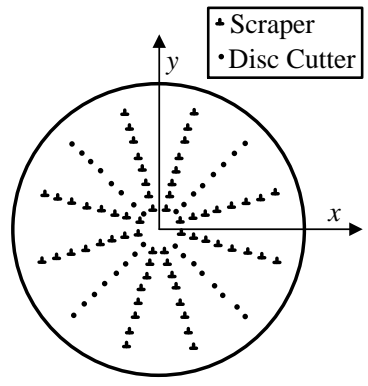

(b)

Fig. 3. (a) The cutter head of some shield tunneling machine; (b) A schematic diagram for the arrangement of the disc cutters and scrapers

and the corresponding torque can be derived as

$$
\begin{aligned}
& M_{x}^{\mathrm{pen}}=\sum_{j=1}^{64}\left[\frac{c_{j}^{\mathrm{coh}} s^{\mathrm{scp}}}{B_{j}^{\mathrm{scp}}}\left(\cos \theta^{\mathrm{scp}}-\mu_{j}^{\mathrm{scp}} \sin \theta^{\mathrm{scp}}\right)\right] \cdot l_{j y}+\sum_{k=1}^{20}\left[\lambda^{\mathrm{disc}} Q_{k}^{\mathrm{disc}} \frac{d^{\mathrm{disc}} w^{\mathrm{disc}} \theta^{\mathrm{disc}}}{2(\psi+1)} \cos \frac{\theta^{\mathrm{disc}}}{2}\right] \cdot l_{k y} \\
& M_{y}^{\mathrm{pen}}=\sum_{j=1}^{64}\left[\frac{c_{j}^{\mathrm{coh}} s^{\mathrm{scp}}}{B_{j}^{s \mathrm{sp}}}\left(\cos \theta^{\mathrm{scp}}-\mu_{j}^{\mathrm{scp}} \sin \theta^{\mathrm{scp}}\right)\right] \cdot l_{j x}+\sum_{k=1}^{20}\left[\lambda^{\mathrm{disc}} Q_{k}^{\mathrm{disc}} \frac{d^{\mathrm{disc}} w^{\mathrm{disc}} \theta^{\mathrm{disc}}}{2(\psi+1)} \cos \frac{\theta^{\mathrm{disc}}}{2}\right] \cdot l_{k x}
\end{aligned}
$$


where $l_{j x}, l_{j y}$ and $l_{k x}, l_{k y}$ respectively denote the coordinates of the $j_{\text {th }}$ scraper and that of the $k_{\text {th }}$ disc cutter; the area of the fracture surface $s^{\mathrm{scp}}=w^{\mathrm{scp}} \cdot h^{\mathrm{scp}} \cdot \tan \theta^{\mathrm{sp}}, w^{\mathrm{spp}}$ and $h^{\mathrm{scp}}$ respectively denote the width and the penetration depth of the scraper, $\alpha^{\mathrm{scp}}$ and $\theta^{\text {sp }}$ respectively denote the angle between the fracture surface and the cutting surface and that between the fracture surface and the moving direction of the scraper; $\lambda^{\text {disc }}$ denotes a dimensionless coefficient, $d^{\text {disc }}$ and $w^{\text {disc }}$ respectively denote the diameter and the width of the disc cutter, $\theta^{\text {disc }}=\arccos \left(1-2 h^{\text {disc }} / d^{\text {disc }}\right)$ denotes the contact angle between the blade and the earth, $h^{\text {disc }}$ denotes the penetration depth of the disc cutter, $l^{\text {disc }}$ denotes the distance between neighboring disc cutters; $c_{j}^{\text {coh }}$ denotes the cohesive strength on the fracture surface, $\mu_{j}^{\text {scp }}$ and $\mu_{j}^{\mathrm{R}}$ respectively denote the friction coefficient between the scraper and the earth and that within the earth, $\sigma_{k}^{\mathrm{c}}$ and $\sigma_{k}^{\mathrm{t}}$ respectively denote the compressive strength and the tensile strength of the earth.

According to existing stratum exploration experiences, composite strata with soft upper stratum and hard lower stratum is one of the most typical strata in tunneling projects (Fig. 4). Its characteristics can be parameterized with a combination of angles $(\varphi, \xi)$, where $\varphi \in(-\pi, \pi)$ denotes the starting angle of some stratum while $\xi \in(0, \pi)$ denotes the central angle of each stratum. Moreover, the rotation of cutter head can result in the variation of the strata that a cutter contacts. Thus, on the basis of the position relationship between points and lines in Plane Geometry, a decisive factor $D R T$ is defined to judge a cutter's relative position to the bound between the upper and lower strata as

where $A_{L}=-[\sin (\varphi+\xi)-\sin \varphi], \quad B_{L}=\cos (\varphi+\xi)-\cos \varphi \quad$ and $\quad C_{L}=R_{\mathrm{sh}} \cdot \sin \xi \quad$ denote the essential coefficients for the dividing line equation, $\left(x^{\text {cut }}, y^{\text {cut }}\right)$ denotes the coordinate of the cutter and $R_{\mathrm{sh}}$ denotes the radius of the cutter head.

Obviously, the characteristics of the earth surrounding the cutter will vary with the changing position of the cutter. When the cutter is above the dividing line and $D R T>0$, values for soft soil will be assigned to the characteristic parameters of the surrounding earth; when the cutter is below the dividing line and $D R T<0$, those for 
hard rock will be assigned to the characteristic parameters of the surrounding earth. Due to the uncertainty of the surrounding earth when the cutter is just on the dividing line and $D R T=0$, the characteristic parameters of the surrounding earth under this condition should take values between those for soft layer and those for hard layer. Assuming that both the soft layer and the hard layer will act on the cutter for periods of same length, we can deem that the arithmetic average of the values for soft and hard layer is suitable to be assigned to the characteristic parameters of the surrounding stratum.

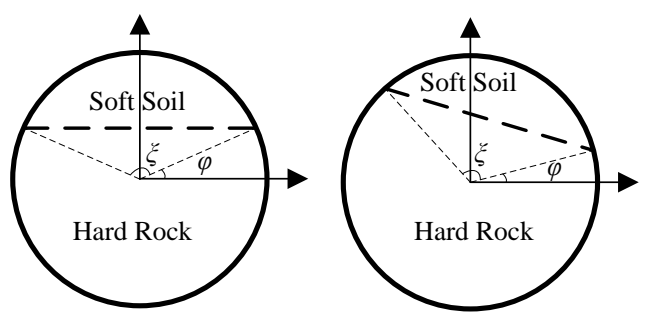

Fig. 4. Schematic diagrams of typical composite stratum

According to the equation for calculating the penetration resistance, $c_{j}^{\mathrm{coh}}, \mu_{j}^{\mathrm{scp}}$, $\mu_{j}^{\mathrm{R}}, \sigma_{k}^{\mathrm{c}}$ and $\sigma_{k}^{\mathrm{t}}$ are the 5 characteristic parameters of the surrounding earth varying with the changing position of the cutter. For the purpose of simplification, a symbol $P R M$ is defined to represents an arbitrary parameter of them. Thus, based on the above analysis on the value selection, each characteristic parameter can be calculated with the following equation

$$
P R M=\frac{P R M_{s}+P R M_{h}}{2}+D R T \cdot \frac{P R M_{s}-P R M_{h}}{2}
$$

where $P R M_{s}$ and $P R M_{h}$ represent the value of characteristic parameter for the soft layer and that for the hard layer respectively.

\subsection{Analysis on the earth pressure of the head face}

The earth pressure normal to the end face of cutter head can be calculated using the equations given in [19] as

$$
F^{\mathrm{ep}}=\pi R_{\mathrm{sh}}^{2} K \gamma_{\mathrm{s}}\left(1-\alpha_{0}\right)\left(h_{0}+R_{\mathrm{sh}}\right)
$$

where $K$ denotes the equivalent coefficient of the horizontal earth pressure, $\gamma_{\mathrm{s}}$ denotes the equivalent weight density of the earth, $\alpha_{0}$ denotes the cutter head opening ratio, $h_{0}$ denotes the height of the collapsing earth derived with Terzaghi 
Theory [25]. In addition, due to the fact that the earth pressure of some point on the end face differs from that of another, the earth pressure will result in a torque about $x$ axis that can be derived as

$$
M_{x}^{\mathrm{ep}}=-\frac{1}{4} \pi R_{\mathrm{sh}}^{4} K \gamma_{\mathrm{s}}\left(1-\alpha_{0}\right)
$$

\subsection{Analysis on the frictional force}

Based on the formulae for the friction of an infinitesimal area of the shield given in [25], the fiction from the $p_{\text {th }}$ stratum can be derived using integral as

$$
F_{p}^{\mathrm{frc}}=R_{\mathrm{sh}} L \mu_{p}^{m s} \gamma_{\mathrm{s}}\left(h_{0}+R_{\mathrm{sh}}\right)\left[\frac{1}{2}\left(1+K_{p}\right) \theta_{p}+\frac{1}{4}\left(1-K_{p}\right) \sin 2 \theta_{p}\right]_{\theta_{p}^{\mathrm{down}}}^{\theta_{p}^{\text {up }}}+
$$

$$
R_{\mathrm{sh}}^{2} L \mu_{p}^{m s} \gamma_{\mathrm{s}}\left[K_{p} \cos \theta_{p}+\frac{1}{3}\left(1-K_{p}\right) \cos ^{3} \theta_{p}\right]_{\theta_{p}^{\text {down }}}^{\text {op }_{\mathrm{p}}^{\text {up }}}
$$

where $L$ denotes the length of the shield, $\mu_{p}^{m s}$ denotes the friction coefficient of the shield against the $p_{\text {th }}$ stratum, $K_{p}$ denotes the horizontal earth pressure coefficient of the $p_{\text {th }}$ stratum, $\theta_{p}^{\text {up }}$ and $\theta_{p}^{\text {down }}$ respectively denote the lower and the upper limit of the central angle of the $p_{\text {th }}$ stratum. Similarly, the corresponding torques about $x$ and $y$ axis caused by the friction of the $p_{\text {th }}$ stratum can be derived as

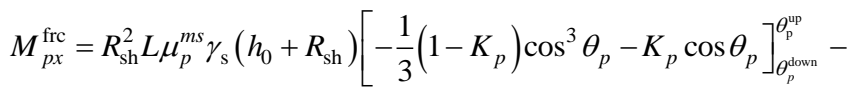

$$
\begin{aligned}
& \frac{1}{2} R_{\mathrm{sh}}^{3} L \mu_{p}^{m s} \gamma_{\mathrm{s}}\left[\frac{1}{4}\left(1+3 K_{p}\right) \theta_{p}-\frac{1}{2} K_{p} \sin 2 \theta_{p}-\frac{1}{16}\left(1-K_{p}\right) \sin 4 \theta_{p}\right]_{\theta_{p}^{\text {town }}}^{\theta_{\mathrm{p}}^{\text {dop }}} \\
& M_{p y}^{\mathrm{frc}}=R_{\mathrm{sh}}^{2} L \mu_{p}^{m s} \gamma_{\mathrm{s}}\left(h_{0}+R_{\mathrm{sh}}\right)\left[\left(-\frac{1}{3}\left(1-K_{p}\right) \sin ^{3} \theta_{p}+\sin \theta_{p}\right)\right]_{\theta_{p}^{\text {down }}}^{\theta_{p \mathrm{p}}^{\text {qu }}} \\
& +\frac{1}{8} R_{\mathrm{ch}}^{3} L \mu_{p}^{m s} \gamma_{\mathrm{s}}\left[\left(1+K_{p}\right) \cos 2 \theta_{p}+\frac{1}{2}\left(1-K_{p}\right) \cos ^{2} 2 \theta_{p}\right]_{\theta_{p}^{\mathrm{dow}}}^{\theta_{\mathrm{p}}^{\mathrm{up}}}
\end{aligned}
$$

For tunneling in composite strata, the total frictional force and torque can finally be calculated by summing the friction and torque from all the $p$ strata.

\section{Case Study on Characteristics of Grouping Strategy}

In tunneling project, hard boulders without detecting by preliminary exploration widely exist along the working path of a shield tunneling machine. They will cause huge impacts onto the cutter head and thus the machine is very likely to deviate from the predetermined trajectory. Because the impacts acting on the cutter head are always reflected as its angular accelerations about the vertical and horizontal axis, the 
compliance ability of the thrust system to these angular accelerations will be studied in this section to give reflections on the performance of the shield tunneling machine under the impacts by using the models derived in the above sections.

A curvilinear tunnel model whose embedded depth and radius are respectively $23.14 \mathrm{~m}$ and $350 \mathrm{~m}$ is used for the case study. All the layers from top to bottom are in turn: $\quad<1>$ Clay, $\quad<5 \mathrm{H}-2>$ Cohesive Soil, $\quad<6 \mathrm{H}>$ Fully-Weathered Granite, $<7 \mathrm{H}>$ Intensively-Weathered Granite, $<8 \mathrm{H}>$ Moderately-Weathered Granite and $<9 \mathrm{H}>$ Slightly-Weathered Granite. Some key geological parameters are listed in Tab. 1 and further need for other parameters can be satisfied by referring to [26]. The tunnel is located in a composite stratum consisting of soft upper layer, i.e. $\langle 8 \mathrm{H}\rangle$ and hard lower layer, i.e. $\langle 9 \mathrm{H}\rangle$.

Tab. 1. Key geologic parameters for some surrounding layers

\begin{tabular}{|c|c|c|c|c|c|}
\hline Mark & $\begin{array}{c}\text { Cohesive } \\
\text { Strength } \\
(\mathbf{M P a})\end{array}$ & $\begin{array}{c}\text { Internal Friction } \\
\text { Angle } \\
\left({ }^{\circ}\right)\end{array}$ & $\begin{array}{c}\text { Earth Pressure } \\
\text { Coefficient }\end{array}$ & $\begin{array}{c}\text { Tensile } \\
\text { Strength } \\
(\mathbf{M P a})\end{array}$ & $\begin{array}{c}\text { Shear } \\
\text { Strength } \\
(\mathbf{M P a})\end{array}$ \\
\hline$\langle 8 \mathrm{H}\rangle$ & 13.69 & 47.7 & 0.30 & 32.80 & 5.50 \\
\hline$\langle 9 \mathrm{H}\rangle$ & 18.63 & 48.2 & 0.19 & 106.50 & 17.80 \\
\hline
\end{tabular}

Elementary parameters of the shield tunneling machine used in this case are listed in Tab. 2. Several typical grouping strategies for its thrust system are shown in Fig. 5, where $\mathrm{A}_{\mathrm{G} q}, \mathrm{~B}_{\mathrm{G} q}, \mathrm{C}_{\mathrm{G} q}, \mathrm{D}_{\mathrm{G} q}$ and $\mathrm{E}_{\mathrm{G} q}(q=1,2,3$ or 4$)$ respectively denote the mark of each group. The arrangement of hydro-cylinders under each strategy is listed in Tab. 3.

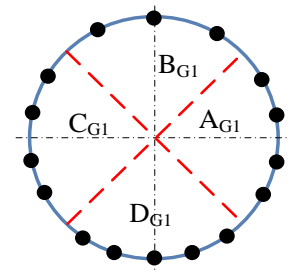

(a)

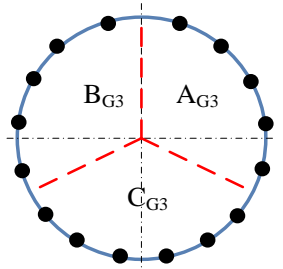

(c)

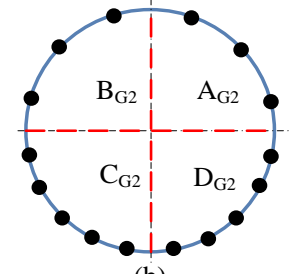

(b)

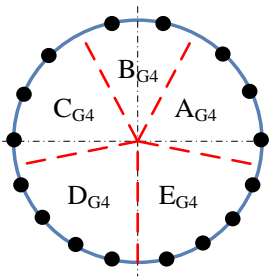

(d)

Fig. 5. Four typical grouping modes: (a) G1: 4-3-4-5 grouping; (b) G2: 3-3-5-5 grouping; (c) 
Tab. 2. Elementary parameters of the shield tunneling machine

\begin{tabular}{|c|c|c|c|}
\hline Diameter & Opening Ratio & Mass of Shield & Length of Shield \\
\hline $6.28 \mathrm{~m}$ & 0.28 & $156.0 \mathrm{t}$ & $1.70 \mathrm{~m}$ \\
\hline Number of Scrapers & \multicolumn{2}{|c|}{ Number of Disc Cutters } & mber of Cylinders \\
\hline 64 & \multicolumn{2}{|c|}{20} & 16 \\
\hline
\end{tabular}

Tab. 3. Arrangement of hydro-cylinders in each group

\begin{tabular}{|c|c|c|c|c|}
\hline Grouping Mode & $\begin{array}{l}\text { Mark of } \\
\text { Groups }\end{array}$ & $\begin{array}{c}\text { Number of } \\
\text { Cylinders }\end{array}$ & $\begin{array}{c}\text { Starting } \\
\text { Phase }\end{array}$ & $\begin{array}{l}\text { Angled } \\
\text { Spacing }\end{array}$ \\
\hline \multirow{4}{*}{ G1 } & $\mathrm{A}_{\mathrm{G} 1}$ & 4 & $-3 \pi / 16$ & $\pi / 8$ \\
\hline & $\mathrm{B}_{\mathrm{G} 1}$ & 3 & $\pi / 3$ & $\pi / 6$ \\
\hline & $\mathrm{C}_{\mathrm{G} 1}$ & 4 & $13 \pi / 16$ & $\pi / 8$ \\
\hline & $\mathrm{D}_{\mathrm{G} 1}$ & 5 & $13 \pi / 10$ & $\pi / 10$ \\
\hline \multirow{4}{*}{$\mathrm{G} 2$} & $\mathrm{~A}_{\mathrm{G} 2}$ & 3 & $\pi / 12$ & $\pi / 8$ \\
\hline & $\mathrm{B}_{\mathrm{G} 2}$ & 3 & $2 \pi / 3$ & $\pi / 8$ \\
\hline & $\mathrm{C}_{\mathrm{G} 2}$ & 5 & $21 \pi / 20$ & $\pi / 10$ \\
\hline & $\mathrm{D}_{\mathrm{G} 2}$ & 5 & $31 \pi / 20$ & $\pi / 10$ \\
\hline \multirow{3}{*}{ G3 } & $\mathrm{A}_{\mathrm{G} 3}$ & 5 & $-\pi / 12$ & $\pi / 8$ \\
\hline & $\mathrm{B}_{\mathrm{G} 3}$ & 5 & $7 \pi / 12$ & $\pi / 8$ \\
\hline & $\mathrm{C}_{\mathrm{G} 3}$ & 6 & $5 \pi / 4$ & $\pi / 10$ \\
\hline \multirow{5}{*}{ G4 } & $\mathrm{A}_{\mathrm{G} 4}$ & 3 & $\pi / 24$ & $\pi / 6$ \\
\hline & $\mathrm{B}_{\mathrm{G} 4}$ & 2 & $11 \pi / 24$ & $\pi / 12$ \\
\hline & $\mathrm{C}_{\mathrm{G} 4}$ & 3 & $5 \pi / 8$ & $\pi / 6$ \\
\hline & $\mathrm{D}_{\mathrm{G} 4}$ & 4 & $25 \pi / 24$ & $\pi / 8$ \\
\hline & $\mathrm{E}_{\mathrm{G} 4}$ & 4 & $19 \pi / 12$ & $\pi / 8$ \\
\hline
\end{tabular}

452

\subsection{Performance Evaluation Criterion for the Shield Tunneling Machine}

Providing huge propelling force onto the cutter head, the thrust system of a shield tunneling machine applies great pressure on the rear segments. In tunneling projects, due to the uneven distribution of the thrusts from the hydro-cylinders, segment overload often exists as a direct cause of segment cracking [14, 27]. These cracks will lead to unexpected deformation of segments and hence reduce the capability of the shield machine to adjust orientation. In addition, this kind of distribution is likely to cause uneven wear among the hydro-cylinders, which will increase the cost for maintenance. Therefore, thrust distribution evenness must be 
considered as a primary performance criterion for the grouping strategy optimization of the thrust system.

Based on the standard deviation of the thrusts in all hydro-cylinders, a Thrust Unevenness Index (TUI) $\kappa_{T U I}$ is proposed as a reflection on thrust distribution evenness to evaluate the compliance ability of the shield tunneling machine:

$$
\kappa_{T U I}=\sqrt{\frac{1}{n-1} \sum_{i=1}^{n}\left(\frac{f_{\mathrm{di}}-\overline{f_{\mathrm{d}}}}{\overline{f_{\mathrm{d}}}}\right)}
$$

where $f_{\mathrm{d} i}$ denotes the thrust of the $i_{\text {th }}$ hydro-cylinder that can be calculated using the force transmission model in Sec. 2, $\overline{f_{\mathrm{d}}}$ denotes the sample average of all the thrusts. Obviously, the smaller the $\kappa_{T U I}$ is, the better the thrust distribution evenness is.

\subsection{Grouping characteristics at different stratum ratios}

Fig. 6-9 respectively show the contours of TUI against the variation of angular acceleration about $x$ and $y$ axes when the stratum ratio equals $20 \%, 40 \%, 60 \%$ and $80 \%$. The contours for each grouping mode are shaped as a cluster of quasi-ellipses. At every stratum ratio, from grouping mode $\mathrm{G} 1$ to $\mathrm{G} 4$, the $x$-axis angular acceleration corresponding to the centers of the contours gradually increases from negative to positive, which means G1 mode is the optimum grouping mode for affording negative impacts about $x$ axis while G4 mode the optimum one for positive impacts; on the other hand, the $y$-axis angular acceleration corresponding to the centers of the contours remains almost unchanged near 0 , which means all the grouping modes hardly possess the capability to resist any impact about $y$ axis. The reason for this phenomenon is that from grouping mode G1 to G4, the uneven distribution of hydro-cylinders about $x$ axis makes a reversal while that about $y$ axis never occurs.

Moreover, with comparison of the centers of the contours for each grouping mode at different stratum ratios, it can be summarized that with the increase of the stratum ratio, the $x$-axis angular acceleration corresponding to the centers of the contours shows a trend of shifting positively first and negatively then. This is mainly because with the rising amount of soft soil, the feature of mono-stratum decreases at first and then increases, which will result in an opposite variation trend of the torque from the thrusts. Due to the symmetrical distribution of the strata about $y$ axis, the angular acceleration around it corresponding to the centers of the contours is almost unchanged near 0 . Besides, in addition to the variation of the contour centers, the 
494

495

496

497

498 499

500

501

contours when stratum ratio equals $80 \%$ still show slight contractions, which indicates the ability of the thrust system to resist impacts among all the grouping modes is reduced.
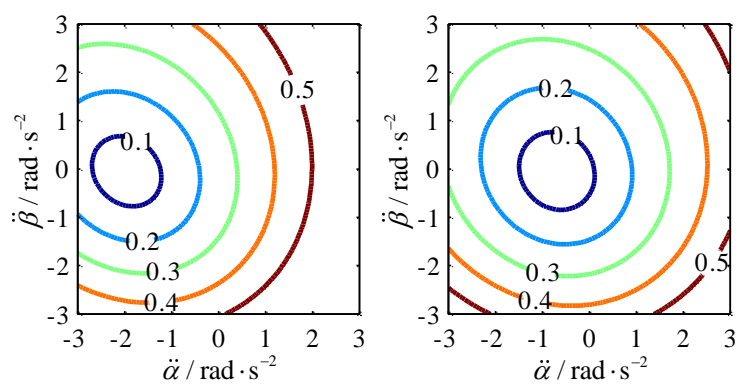

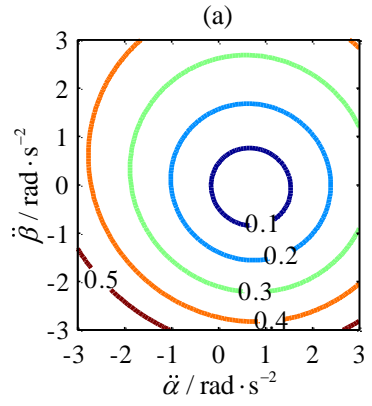

(c)

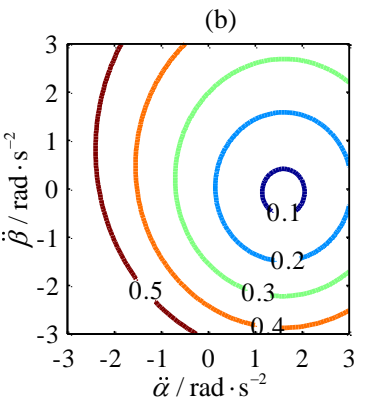

(d)

Fig. 6. Contours of TUI when the stratum ratio equals 20\%: (a) G1 mode; (b) G2 mode; (c) G3 mode; (4) G4 mode
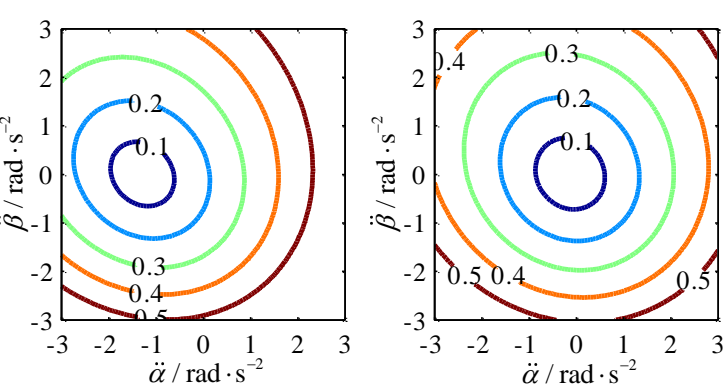

(a)

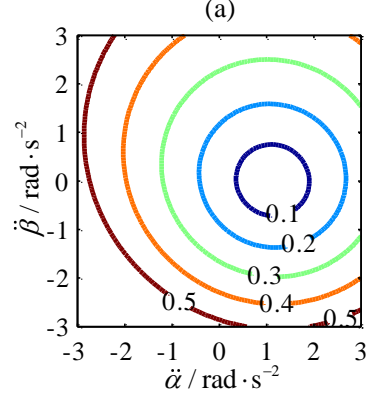

(b)

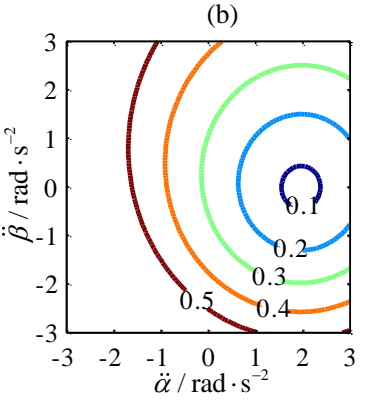

(d)

Fig. 7. Contours of $T U I$ when the stratum ratio equals 40\%: (a) G1 mode; (b) G2 mode; (c) G3 mode; (4) G4 mode 

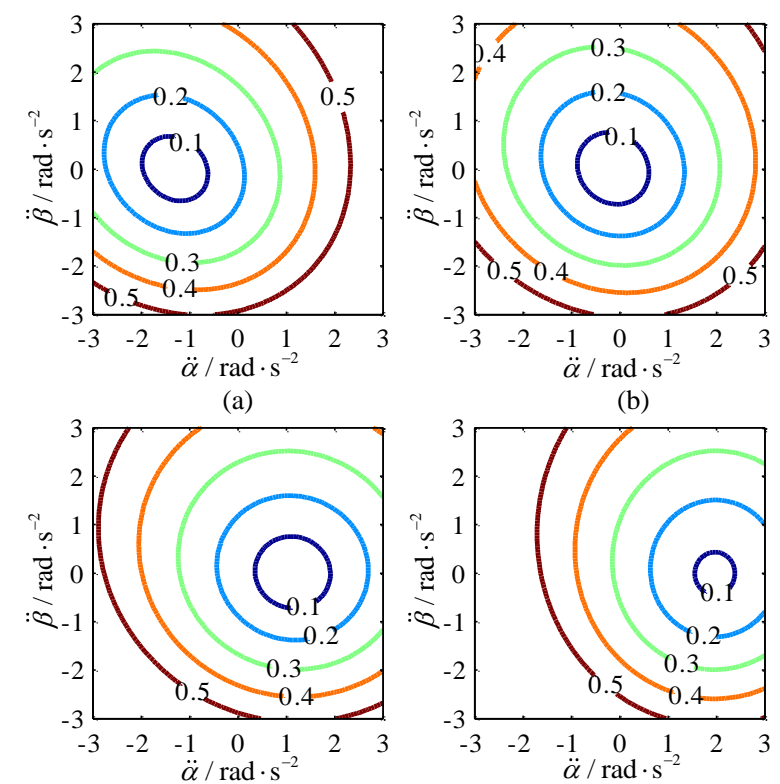

(c)

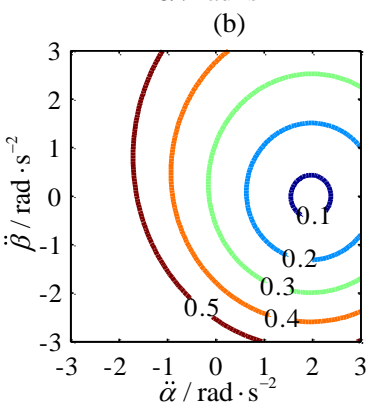

(d)

Fig. 8. Contours of TUI when the stratum ratio equals 60\%: (a) G1 mode; (b) G2 mode; (c) G3 mode; (4) G4 mode
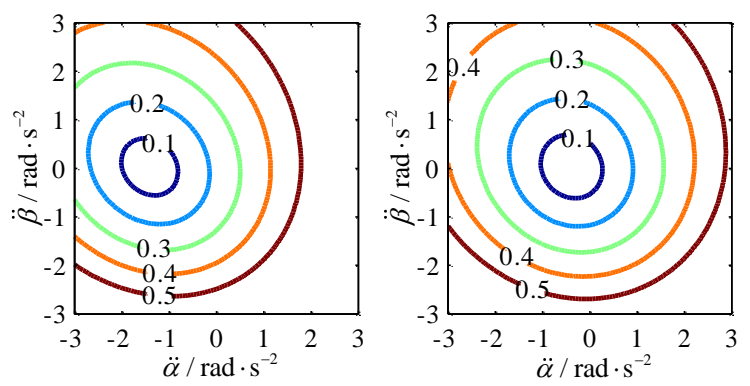

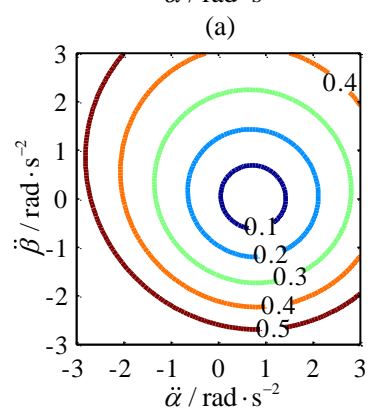

(c)

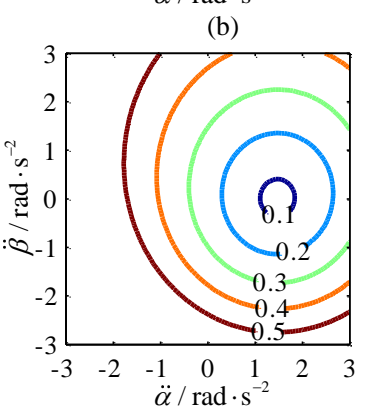

(d)

Fig. 9. Contours of $T U I$ when the stratum ratio equals $80 \%$ : (a) G1 mode; (b) G2 mode; (c)

G3 mode; (4) G4 mode

\subsection{Grouping characteristics at different orientations of the dividing line}

Fig. 10-13 respectively show the contours of TUI against the variation of angular acceleration about $x$ and $y$ axes when the tilting angle of the dividing line equals $\pi / 3$, $\pi / 6,5 \pi / 6$ and $2 \pi / 3$. The stratum ratio here is constant at $40 \%$ for the pulses of the 
thrusts are relatively significant in this situation [28]. For each tilting angle, the centers of the contours of all grouping modes show similar variation trend with that for them in last part. Thus, it can still be concluded that G1 and G4 modes are respectively the optimum grouping mode to resist negative impacts and that to resist positive impacts about $x$ axis, while no grouping mode can afford any impacts about $y$ axis. The findings are exactly the same with those in last part, which verifies the correctness of the former deduction.
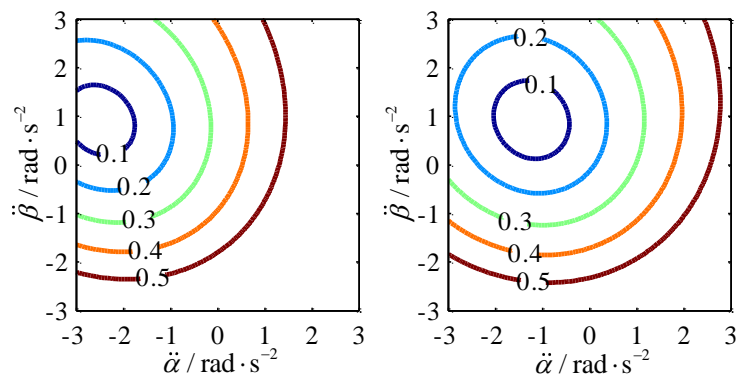

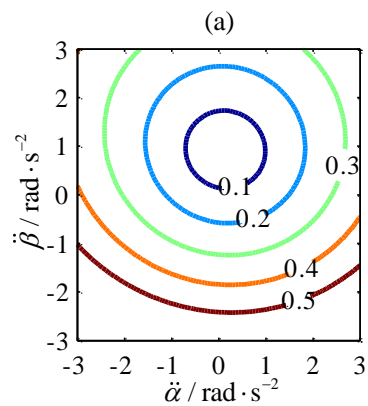

(c)

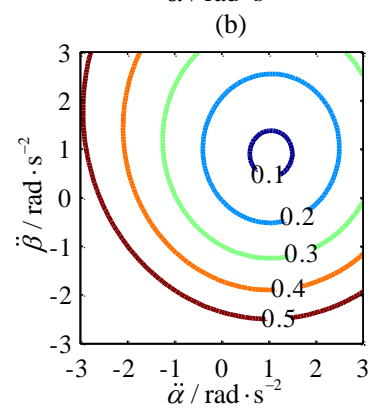

(d)

Fig. 10. Contours of TUI when the dividing line tilts by $\pi / 3$ : (a) G1 mode; (b) G2 mode; (c) G3 mode; (4) G4 mode
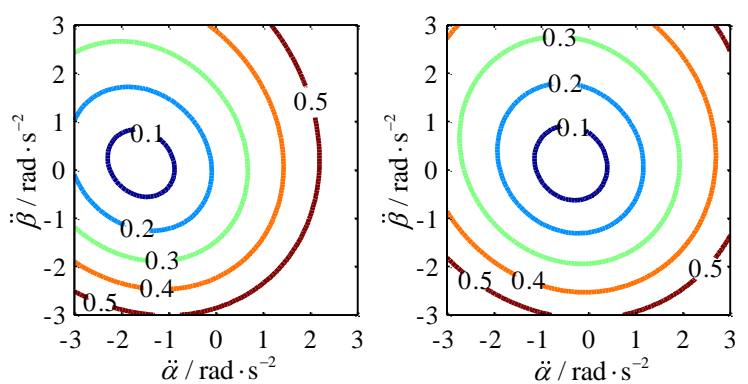

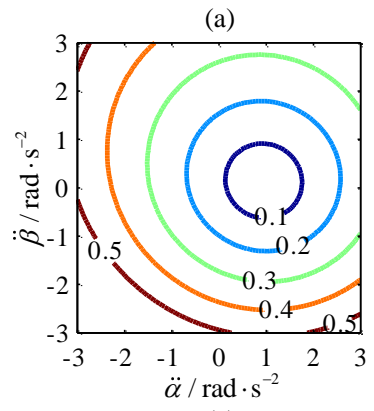

(c)

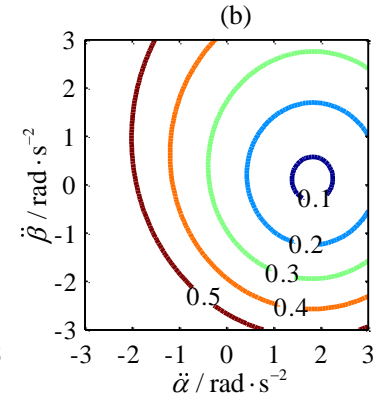

(d) 
Fig. 11. Contours of $T U I$ when the dividing line tilts by $\pi / 6$ : (a) G1 mode; (b) G2 mode; (c) G3 mode; (4) G4 mode

With further comparison on the centers of the contours for each grouping mode at different tilting angles with regard to the contours in Fig. 7, it can be summarized that the $x$-axis angular acceleration corresponding to the center of each contour also shows a trend of shifting positively at first and then negatively with the increase of the tilting angle, while the $y$-axis acceleration corresponding to them always shifts negatively. Supposing $\pi / 3$ and $2 \pi / 3, \pi / 6$ and $5 \pi / 6$ are two symmetric combinations of the tilting angles, of which the sum is $\pi$, the variation for the centers of the contours are almost the same to each combination. That is to say, the variation with the changing tilting angle is axisymmetric. The main causes for the finding are supposed to be: 1) the scalars of the resistance torques about $x$ axis decrease when the tilting angle varies from $\pi / 3$ to 0 and the opposite variation will occur when the tilting angle varies from $\pi$ to $2 \pi / 3 ; 2$ ) the dividing lines are symmetric about $y$ axis under both combinations, which will result in a direction reversal of the resistance torque about $y$ axis without changing about $x$ axis.

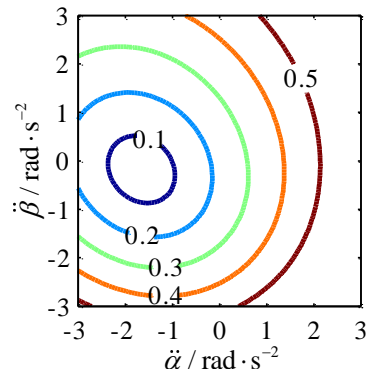

(a)

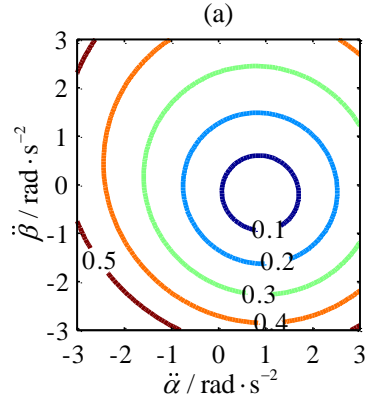

(c)

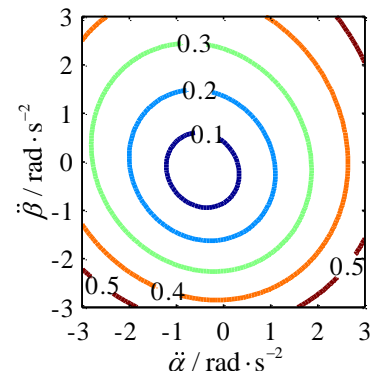

(b)

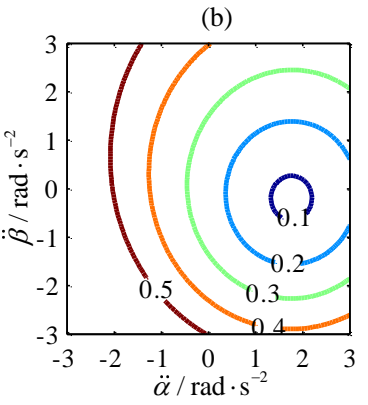

(d)

Fig. 12. Contours of $T U I$ when the dividing line tilts by $5 \pi / 6$ : (a) G1 mode; (b) G2 mode; (c) G3 mode; (4) G4 mode 

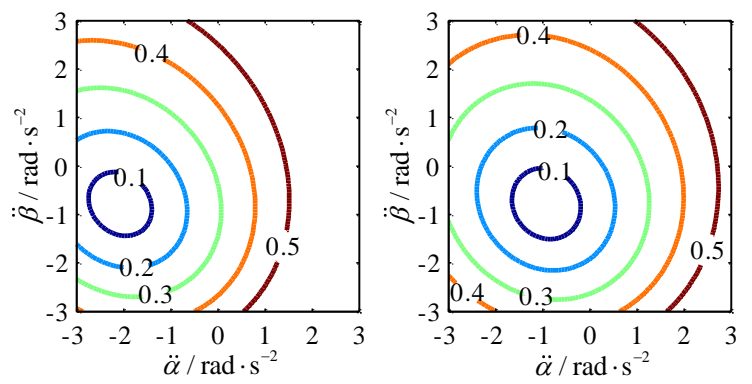

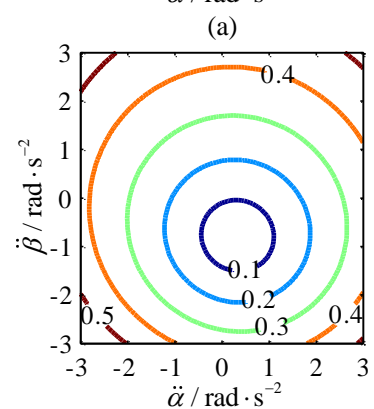

(c)

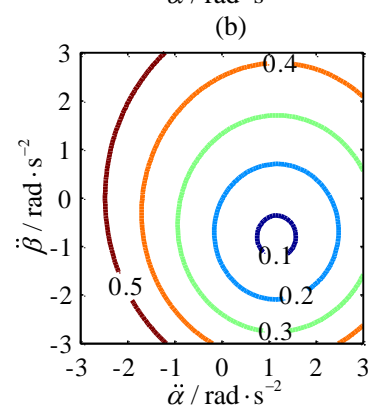

(d)

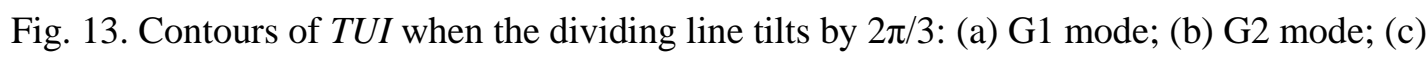
G3 mode; (4) G4 mode

Another finding within these figures is that the variations for the centers of the contours when the tilting angle is between $\pi / 3$ and $\pi / 6$ is greater than those when the tilting angle is between $\pi / 6$ and 0 . This is mainly because the resistance torque is gradually insensitive to the variation of the orientation of the dividing line, when it is close to the horizontal posture. Similar results can be summarized during the opposite interval for the tilting angle due to symmetry.

\section{Conclusions}

In the current paper, a dynamic force transmission model for the thrust system of a shield tunneling machine is proposed to optimize the grouping strategies for the thrust system and investigate the effect of geological characteristics on the performance of the machine. Due to consideration of the actions from the surrounding stratum as a part of mechanism constraints, this model can provide a relatively accurate calculation on thrust forces on each group of hydro-cylinders. Through case study, the evaluation criterion TUI at different stratum ratios and different orientations of the dividing line is analyzed to draw the following conclusions:

1) Under a given condition of surrounding strata, a dynamic model of a thrust system can help establish a relatively accurate calculation of the propelling forces needed in each hydro-cylinder. 
2) For an arbitrary thrust system, the contours of TUIs are a cluster of quasi-ellipses whose center can reveal the most suitable impacts that the shield tunneling machine can afford. These centers vary with the changing grouping modes and hence can be used with specific TUIs to optimize the grouping strategy of the thrust system.

3) In composite strata, where the ratio between soft soil and hard rock or the tilting angle of the dividing line is varying, the adaptability of the thrust system to impacts will show significant change. Therefore, real-time control of the machine is necessary for reducing the offset load to avoid segment cracking.

It is hoped that the findings in this study will give useful instructions to group control of a shield tunneling machine in tunneling projects. However, some limitations must be mentioned here. The equivalent mechanism constraints are not sufficiently accurate to reveal restrictions from surrounding strata. Furthermore, the simplification of a grouped thrust system is only available for the condition when the relative angle between the cutter head and the end face of segment is extremely small. Thus, further research is required to take into consideration variable kinematic constraints from surrounding strata and reasonable equivalence for a grouped thrust system.

\section{Acknowledgements}

This research is jointly supported by the National Science Foundation of China (NSFC) under Grant 51305256 and Grant 11472172, and the National Basic Research Program of China (973 Program) under research grant 2013CB035403, and SMC Morningstar Scholars Program of SJTU.

\section{Appendix}

A. Elements of $\boldsymbol{J}_{\mathrm{Tm}}$ in Eq. (9): 


$$
\left\{\begin{array}{l}
J_{\mathrm{T} m 11}=\sin \beta \\
J_{\mathrm{T} m 2}=0 \\
J_{\mathrm{T} 13}=R_{\mathrm{sh}} \cos \chi \sin \beta+\left(r-l_{\mathrm{p}}\right) \cos \beta \\
J_{\mathrm{T} 21}=-\sin \alpha \cos \beta \\
J_{\mathrm{T} 22}=-R_{\mathrm{sh}} \cos \chi \cos \alpha \sin \beta+R_{\mathrm{sh}} \sin \chi \sin \alpha-\left(r-l_{\mathrm{p}}\right) \cos \alpha \cos \beta \\
J_{\mathrm{T} 23}=-R_{\mathrm{sh}} \cos \chi \sin \alpha \cos \beta+\left(r-l_{\mathrm{p}}\right) \sin \alpha \sin \beta \\
J_{\mathrm{T} 31}=\cos \alpha \cos \beta \\
J_{\mathrm{T} 32}=-R_{\mathrm{sh}} \cos \chi \sin \alpha \sin \beta-R_{\mathrm{sh}} \sin \chi \cos \alpha-\left(r-l_{\mathrm{p}}\right) \sin \alpha \cos \beta \\
J_{\mathrm{T} 333}=R_{\mathrm{sh}} \cos \chi \cos \alpha \cos \beta-\left(r-l_{\mathrm{p}}\right) \cos \alpha \sin \beta
\end{array}\right.
$$

602

B. Elements of $\varepsilon$ in Eq. (9):

604

$$
\left\{\begin{aligned}
\varepsilon_{11}= & 2 \cos \beta \dot{r} \dot{\beta}+\left(R_{\mathrm{sh}} \cos \chi \cos \beta-\left(r-l_{\mathrm{p}}\right) \sin \beta\right) \dot{\beta}^{2} \\
\varepsilon_{21}= & -2 \cos \alpha \cos \beta \dot{r} \dot{\alpha}+2 \sin \alpha \sin \beta \dot{r} \dot{\beta}- \\
& 2\left(R_{\mathrm{sh}} \cos \chi \cos \alpha \cos \beta-\left(r-l_{\mathrm{p}}\right) \cos \alpha \sin \beta\right) \dot{\alpha} \dot{\beta}+ \\
& \left(R_{\mathrm{sh}} \sin \chi \cos \alpha+R_{\mathrm{sh}} \cos \chi \sin \alpha \sin \beta+\left(r-l_{\mathrm{p}}\right) \sin \alpha \cos \beta\right) \dot{\alpha}^{2}+ \\
& \left(R_{\mathrm{sh}} \cos \chi \sin \alpha \sin \beta+\left(r-l_{\mathrm{p}}\right) \sin \alpha \cos \beta\right) \dot{\beta}^{2} \\
\varepsilon_{31}= & -2 \sin \alpha \cos \beta \dot{r} \dot{\alpha}-2 \cos \alpha \sin \beta \dot{r} \dot{\beta}- \\
& 2\left(R_{\mathrm{sh}} \cos \chi \sin \alpha \cos \beta+\left(r-l_{\mathrm{p}}\right) \sin \alpha \sin \beta\right) \dot{\alpha} \dot{\beta}+ \\
& \left(R_{\mathrm{sh}} \sin \chi \sin \alpha-R_{\mathrm{sh}} \cos \chi \cos \alpha \sin \beta-\left(r-l_{\mathrm{p}}\right) \cos \alpha \cos \beta\right) \dot{\alpha}^{2}- \\
& \left(R_{\mathrm{sh}} \cos \chi \cos \alpha \sin \beta+\left(r-l_{\mathrm{p}}\right) \cos \alpha \cos \beta\right) \dot{\beta}^{2}
\end{aligned}\right.
$$

605

606

607

C. Elements of $\boldsymbol{l}_{i}$ in Eq. (10):

$$
\left\{\begin{aligned}
l_{i x}= & \left(r-l_{\mathrm{p}}\right) \sin \beta+R_{\mathrm{sh}}\left(\cos \chi-\cos \varphi_{i}\right)+R_{\mathrm{sh}}\left(\cos \varphi_{i}-\cos \chi\right) \cos \beta \\
l_{i y}= & -\left(r-l_{\mathrm{p}}\right) \sin \alpha \cos \beta+R_{\mathrm{sh}}\left(\sin \chi-\sin \varphi_{i}\right)+ \\
& R_{\mathrm{sh}}\left(\cos \varphi_{i}-\cos \chi\right) \sin \alpha \sin \beta+R_{\mathrm{sh}}\left(\sin \varphi_{i}-\sin \chi\right) \cos \alpha \\
l_{i z} & l_{\mathrm{p}}+\left(r-l_{\mathrm{p}}\right) \cos \alpha \cos \beta-R_{\mathrm{sh}}\left(\cos \varphi_{i}-\cos \chi\right) \cos \alpha \sin \beta+ \\
& R_{\mathrm{sh}}\left(\sin \varphi_{i}-\sin \chi\right) \sin \alpha
\end{aligned}\right.
$$

608

609 D. Elements of $\boldsymbol{K}(\boldsymbol{q}, \dot{\boldsymbol{q}})$ in Eq. (25) and (28): 


$$
\left\{\begin{aligned}
K_{11}= & 2 m_{\mathrm{m}} \cos \beta \dot{r} \dot{\beta}+m_{\mathrm{m}}\left(R_{\mathrm{sh}} \cos \chi \cos \beta-\left(r-l_{\mathrm{p}}\right) \sin \beta\right) \dot{\beta}^{2} \\
K_{21}= & -2 m_{\mathrm{m}} \cos \alpha \cos \beta \dot{r} \dot{\alpha}+2 m_{\mathrm{m}} \sin \alpha \sin \beta \dot{r} \dot{\beta}- \\
& 2 m_{\mathrm{m}}\left(R_{\mathrm{sh}} \cos \chi \cos \alpha \cos \beta-\left(r-l_{\mathrm{p}}\right) \cos \alpha \sin \beta\right) \dot{\alpha} \dot{\beta}+ \\
& m_{\mathrm{m}}\left(R_{\mathrm{sh}} \sin \chi \cos \alpha+R_{\mathrm{sh}} \cos \chi \sin \alpha \sin \beta+\right. \\
& \left.\left(r-l_{\mathrm{p}}\right) \sin \alpha \cos \beta\right) \dot{\alpha}^{2}+ \\
& m_{\mathrm{m}}\left(R_{\mathrm{sh}} \cos \chi \sin \alpha \sin \beta+\left(r-l_{\mathrm{p}}\right) \sin \alpha \cos \beta\right) \dot{\beta}^{2} \\
K_{31}= & -2 m_{\mathrm{m}} \sin \alpha \cos \beta \dot{r} \dot{\alpha}-2 m_{\mathrm{m}} \cos \alpha \sin \beta \dot{r} \dot{\beta}- \\
& 2 m_{\mathrm{m}}\left(R_{\mathrm{sh}} \cos \chi \sin \alpha \cos \beta+\left(r-l_{\mathrm{p}}\right) \sin \alpha \sin \beta\right) \dot{\alpha} \dot{\beta}+ \\
& m_{\mathrm{m}}\left(R_{\mathrm{sh}} \sin \chi \sin \alpha-R_{\mathrm{sh}} \cos \chi \cos \alpha \sin \beta-\right. \\
& \left.\left(r-l_{\mathrm{p}}\right) \cos \alpha \cos \beta\right) \dot{\alpha}^{2}- \\
& m_{\mathrm{m}}\left(R_{\mathrm{sh}} \cos \chi \cos \alpha \sin \beta+\left(r-l_{\mathrm{p}}\right) \cos \alpha \cos \beta\right) \dot{\beta}^{2} \\
K_{41}= & -\left(I_{\mathrm{m} 12} \sin \alpha-I_{\mathrm{m} 13} \cos \alpha+I_{\mathrm{m} 21} \sin \alpha-\right. \\
& \left.I_{\mathrm{m} 32} \cos { }^{2} \alpha-I_{\mathrm{m} 33} \sin \alpha \cos \alpha\right) \dot{\alpha} \dot{\beta}+I_{\mathrm{m} 31} \cos \alpha \dot{\alpha}^{2}- \\
& \left(I_{\mathrm{m} 22} \sin \alpha \cos \alpha+I_{\mathrm{m} 23} \sin { }^{2} \alpha\right) \dot{\beta}^{2} \\
K_{51}= & \left(I_{\mathrm{m} 11} \sin \alpha-I_{\mathrm{m} 22} \sin \alpha+I_{\mathrm{m} 23} \cos \alpha-\right. \\
& \left.I_{\mathrm{m} 32} \cos \alpha-I_{\mathrm{m} 33} \sin \alpha\right) \dot{\alpha} \dot{\beta}-I_{\mathrm{m} 31} \dot{\alpha}^{2}+ \\
& \left(I_{\mathrm{m} 12} \sin \alpha \cos \alpha+I_{\mathrm{m} 13} \sin { }^{2} \alpha\right) \dot{\beta}^{2} \\
K_{61}= & -\left(I_{\mathrm{m} 12} \cos 2 \alpha+I_{\mathrm{m} 13} \sin \alpha \cos \alpha-I_{\mathrm{m} 22} \cos \alpha-\right. \\
& \left.I_{\mathrm{m} 23} \sin \alpha+I_{\mathrm{m} 32} \sin \alpha-I_{\mathrm{m} 33} \cos \alpha\right) \dot{\alpha} \dot{\beta}- \\
& \left(I_{\mathrm{m} 11} \cos \alpha-I_{\mathrm{m} 21}\right) \dot{\alpha}^{2}
\end{aligned}\right.
$$

611

$$
\left\{\begin{aligned}
B_{i 11}= & \sin \beta\left(\sin \phi_{i} \cos \eta_{i} \cos \varphi_{i}+\sin \eta_{i} \sin \varphi_{i}\right)- \\
& \sin \alpha \cos \beta\left(\sin \phi_{i} \cos \eta_{i} \sin \varphi_{i}-\sin \eta_{i} \cos \varphi_{i}\right)+ \\
& \cos \alpha \cos \beta \cos \phi_{i} \cos \eta_{i} \\
B_{i 21}= & -\left[R _ { \mathrm { sh } } \left(\left(\cos \chi-\cos \theta_{\mathrm{P} i}\right) \cos \alpha \sin \beta-\right.\right. \\
& \left.\left.\left(\sin \chi-\sin \theta_{\mathrm{P} i}\right) \sin \alpha\right)+\left(r-l_{\mathrm{p}}\right) \cos \alpha \cos \beta\right] \\
& \left(\sin \phi_{i} \cos \eta_{i} \sin \varphi_{i}-\sin \eta_{i} \cos \varphi_{i}\right)- \\
& {\left[R _ { \mathrm { sh } } \left(\left(\cos \chi-\cos \theta_{\mathrm{P} i}\right) \sin \alpha \sin \beta+\right.\right.} \\
& \left.\left(\sin \chi-\sin \theta_{\mathrm{P} i}\right) \cos \alpha\right)+ \\
& \left.\left(r-l_{\mathrm{p}}\right) \sin \alpha \cos \beta\right] \cos \phi_{i} \cos \eta_{i} \\
B_{i 31} & {\left[R_{\mathrm{sh}}\left(\cos \chi-\cos \theta_{\mathrm{P} i}\right) \sin \beta+\right.} \\
& \left.\left(r-l_{\mathrm{p}}\right) \cos \beta\right]\left(\sin \phi_{i} \cos \eta_{i} \cos \varphi_{i}+\sin \eta_{i} \sin \varphi_{i}\right)- \\
& {\left[R_{\mathrm{sh}}\left(\cos \chi-R_{\mathrm{sh}} \cos \theta_{\mathrm{P} i}\right) \sin \alpha \cos \beta-\right.} \\
& \left.\left(r-l_{\mathrm{p}}\right) \sin \alpha \sin \beta\right]\left(\sin \phi_{i} \cos \eta_{i} \sin \varphi_{i}-\sin \eta_{i} \cos \varphi_{i}\right)+ \\
& {\left[R_{\mathrm{sh}}\left(\cos \chi-\cos \theta_{\mathrm{P} i}\right) \cos \alpha \cos \beta-\right.} \\
& \left.\left(r-l_{\mathrm{p}}\right) \cos \alpha \sin \beta\right] \cos \phi_{i} \cos \eta_{i}
\end{aligned}\right.
$$




\section{References}

[1] Koyama Y., 2003. Present status and technology of shield tunneling method in Japan. Tunneling and Underground Space Technology, 18(2), 145-159.

[2] Zhao Y., 2013. Compliance Analysis and Design of the Mechanical System in shield Tunneling Machines (in Chinese). Shanghai Jiao Tong University.

[3] Hassanpour J., Rostami J., Khamehchiyan M., Bruland A., 2009. Developing new equations for TBM performance prediction in carbonate-argillaceous rocks: a case history of Nowsood water conveyance tunnel. Geomechanics and Geoengineering 4(4), 287-297.

[4] Hamidi J.K., Shahriar K., Rezai B., Rostami J., 2010. Performance prediction of hard rock TBM using Rock Mass Rating (RMR) system. Tunneling And Underground Space Technology 25(4), 333-345.

[5] Hassanpour J., Rostami J., Zhao J., 2011. A new hard rock TBM performance prediction model for project planning. Tunneling And Underground Space Technology 26(5), 595-603.

[6] Copur H., Aydin H., Bilgin H., Balci C., Tumac D., Dayanc C., 2014. Predicting performance of EPB TBMs by using a stochastic model implemented into a deterministic model. Tunneling and Underground Space Technology 42, 1-14.

[7] Yang H.Y., Shi H., Gong G.F., Hu G.L., 2009. Electro-hydraulic proportional control of thrust system for shield tunneling machine. Automation in Construction 18(7), 950-956.

[8] Liu H.J., Wang J.C., Zhang L.W., Fei L., Zhao G.L., 2014. Dynamic Modeling and Trajectory Tracking Control of Tunnel Boring Machine. In Proceedings of the 26th Chinese Control and Decision Conference, 4560-4565.

[9] Li X.H., Yu H.B., Yuan M.Z., Wang J., Yin Y., 2010. Dynamic Modeling and Analysis of Shield TBM Cutterhead Driving System. Journal of Dynamic Systems, Measurement and Control 132(4).

[10] Liu Z.P., Chen G.L., Yu H.D., Lai X.M., 2009. The Compliance Design on Thrust System of Shields Excavating in Various Geologic Conditions. ICIRA 2009, LNAI 5928, 297-306.

[11] Deng Y.C., Guo W.Z., Gao F., 2010. Equivalent Mechanism-based Modeling for Grouping Performance Analysis of the Thrust System of Shield Machines (in Chinese). Journal of Mechanical Engineering 46(13), 122-127.

[12] Deng K.S., Tang X.Q., Wang L.P., Chen X., 2011. Force transmission characteristics for the non-equidistant arrangement thrust systems of shield tunneling machines. Automation in Construction 20(5), 588-595.

[13] Deng K.S., Tang X.Q., Wang L.P., Chen X., 2011. Research on characteristics of 
deformation in thrust system for EPB shield machines. Tunneling and Underground Space Technology 26(1), 15-21.

[14] Deng K.S., Huang J.L., Wang H.G., 2015. Layout optimization of non-equidistant arrangement for thrust systems in shield machines. Automation in Construction 49, 135-141.

[15] Sugimoto M., Sramoon A., 2002. Theoretical Model of Shield Behavior During Excavation. I: Theory. Journal of Geotechnical and Geoenvironmental Engineering 128(2), 138-155.

[16] Sramoon A., Sugimoto M., Kayukawa K., 2002. Theoretical Model of Shield Behavior During Excavation. II: Application. Journal of Geotechnical \& Geoenvironmental Engineering 128(2), 156-165.

[17] Wang L.T., Gong G.F., Shi H., Yang H.Y., 2012. Modeling and analysis of thrust force for EPB shield tunneling machine. Automation in Construction 27, 138-146.

[18] Ates U., Bilgin U., Copur H., 2014. Estimating torque, thrust and other design parameters of different type TBMs with some criticism to TBMs used in Turkish tunneling projects. Tunneling and Underground Space Technology 40, 46-63.

[19] Zhang Q., Huang T., Huang G.Y., Cai Z.X., Kang Y.L., 2013. Theoretical model for loads prediction on shield tunneling machine with consideration of soil-rock interbedded ground. Science China Technological Sciences 56(9), 2259-2267.

[20] Zhang Q., Qu C., Cai Z., Kang Y., Huang T., 2014. Modeling of the thrust and torque acting on shield machines during tunneling. Automation in Construction 40(4), 60-67.

[21] Schiehlen W., 1997. Multi-Body System Dynamics: Roots and Perspectives. Multibody System Dynamics 1, 149-188.

[22] Orin E., Oh Y., 1981. Control of Force Distribution in Robotic Mechanisms Containing Closed Kinematic Chains. Journal of Dynamic Systems, Measurement, and Control 102, 134-141.

[23] Zeng X.X., Yu H.D., Zhang K.Z., Wang H., 2010. Bending moments study on the cutterheads of shield machines excavating in composition geologic strata (in Chinese). Journal of Shanghai Jiao Tong University 44(1), 51-55.

[24] Rostami J., Ozdemir L., Nilson B., 1996. Comparison between CSM and NTH Hard Rock TBM Performance Prediction Models. In Proceedings of Institute of Shaft Drilling Technology (ISDT) Annual Technical Conference 11.

[25] Guan H.S., 2007. Study on the Calculating Models of Key Parameters and Mechanical Behavior of the EPB Shield Machine (in Chinese). Southwest Jiao Tong University.

[26] Zhu W.Z., Ju S.J., Shi H.O., 2007. Guangzhou Metro Line 3 Shield Tunneling Construction Technology (in Chinese). Jinan University Press.

[27] Chen J.S., Mo H.H., 2009. Numerical study on crack problems in segments of shield 

tunnel using finite element method. Tunneling and Underground Space Technology 24(1), 91-102.

[28] Shao X., Yu H.D., Zhang K.Z., Wang H., 2011. Grouping Strategy Research on Shield Machine Thrust System Excavating in Compound Geology (in Chinese). Machine Design and Research 27(1), 5-9. 